\title{
Die Polexit-Debatte
}

\section{ANALYSE}

Reale Gefahr oder Scheindebatte?

Zum Wirklichkeitsgehalt eines drohenden Polexit

Irene Hahn-Fuhr, Zentrum Liberale Moderne, Berlin, und Gert Röhrborn,

Warschau

DOKUMENTATION

Rede von Ministerpräsident Mateusz Morawiecki vor dem Europäischen

Parlament, 19. Oktober 2021 [Auszüge]

STATISTIK

Veränderungen des Bruttoinlandsprodukts Polens pro Kopf seit dem Beitritt Polens zur Europäischen Union

UMFRAGEN

Einstellungen zur Europäischen Union

CHRONIK

01. - 14. Februar 2022

Deutsches

Polen-Institut
Forschungsstelle Osteuropa an der Universität Bremen
Leibniz-Institut für

Agrarentwicklung in

Transformationsökonomien
Leibniz-Institut für Ost- und Südosteuropa-

forschung 


\section{Reale Gefahr oder Scheindebatte? Zum Wirklichkeitsgehalt eines drohenden Polexit}

Irene Hahn-Fuhr, Zentrum Liberale Moderne, Berlin, und Gert Röhrborn, Warschau

DOI: $10.31205 /$ PA.288.01

\section{Zusammenfassung}

Seit der Auseinandersetzung um die Entscheidung des polnischen Verfassungstribunals zum Vorrang polnischen Rechts über EU-Recht im Oktober 2021 wird sowohl innerhalb als auch außerhalb Polens verstärkt über die mögliche Gefahr eines Austritts unseres Nachbarlands aus der EU spekuliert. Nach Jahren der Auseinandersetzung mit EU-Kommission, EU-Parlament und Europäischem Gerichtshof stellt das kompromisslose Auftreten der polnischen Regierung die Institutionen und Partnerstaaten vor die Herausforderung, wie in den Fragen um Gewaltenteilung und unabhängige Justiz mit ihrem östlichen Mitgliedsstaat weiter umgegangen werden sollte. Auch die Bundesregierung ist konkret gefordert, die in der Ära Merkel verfolgte Politik der vorsichtigen Beschwichtigung grundlegend zu überdenken. Dafür ist aber zunächst einmal zu klären, welche Interessen hinter den unterschiedlichen Argumentationslinien im Diskurs der verschiedenen polnischen Parteien und Akteure liegen, wenn sie mit dem politischen Kampfbegriff „Polexit« hantieren.

$\mathrm{N}$ ach mehr als 15 Jahren profitabler Mitgliedschaft Polens in der Europäischen Union wird neuerdings über einen »Polexit" gemutmaßt - also über einen möglichen Austritt unseres östlichen Nachbarn aus der europäischen Staatengemeinschaft, die neben der NATO seit dem Ende des Kommunismus ein unverrückbarer Teil der polnischen Staatsraison gewesen ist. Doch angesichts der Eskalation zwischen der polnischen Regierung der Partei Recht und Gerechtigkeit (Prawo i Sprawiedliwość - PiS) und den EU-Institutionen in der Auseinandersetzung um die Umsetzung des Rechtsstaatlichkeitsprinzips ist dieses Stichwort schlagartig zur Beschreibung einer akuten Bedrohungslage avanciert, wie auch Reinhold Vetter in seinem Beitrag für die Polen-Analysen (Nr. 285, https://www.laender-analysen. de/polen-analysen/285/polexit-durch-die-hintertuerpolnische-regierungspolitik-und-europaeische-themen/) dargelegt hat. Im Unterschied zu Vetter, der eher westliche Akteure als Schöpfer der Polexit-These vermutet, soll in der vorliegenden Ausgabe näher beleuchtet werden, wo die Quellen der Debatte im Lande selbst zu suchen sind.

Das Urteil des polnischen Verfassungstribunals (Trybunat Konstytucyjny - TK) vom 7. Oktober 2021 ist von manchen Kreisen im In- und Ausland als erster (de jure) Schritt auf dem Weg Polens aus der EU hinaus wahrgenommen worden. Es wirft die Frage auf, ob es sich die PiS-Regierung mit Verweis auf den angeblichen Vorrang polnischen Rechts erlauben würde, in einer abgespeckten Form in der EU zu funktionieren - also sich schlicht nicht an alle Vorgaben aus den Verträgen zu halten, indem sie einfach jedes ihr nicht genehme Urteil des Europäischen Gerichtshofes (EuGH) über das von ihr kontrollierte Verfassungstribunal für ungültig erklären lässt. Am Ende dieses Weges einer so eklatanten Missachtung der EU-Rechtsordnung, so die Befürchtung, könnte dann mittelfristig ein tatsächlicher Austritt aus der Staatengemeinschaft stehen. Dies hat nicht nur in den Medien, sondern auch in der polnischen Gesellschaft deutliche Spuren hinterlassen. Die Zahl derjenigen, die einen Polexit als reales Szenario betrachten, stieg laut einer Umfrage von United Surveys im Laufe weniger Wochen von 30 auf 42 Prozent. Angenommen, sie hätten Recht: Wie würde ein Austritt Polens aus der EU formal überhaupt vonstattengehen können?

\section{Ein Blick auf die rechtlichen Grundlagen}

Die Verfassung der Republik Polen regelt in den Artikeln 89 bis 91 ausführlich den Beitritt zu und die Ausführung von internationalen Verträgen einschließlich deren Vorrang vor nationalen Gesetzen im Falle der Unvereinbarkeit mit letzteren. Eine entsprechende Kündigung wird hingegen lediglich als möglich erwähnt. Die entsprechenden Modalitäten sind im Gesetz über internationale Verträge vom 14. April 2000 festgelegt. Dessen Art. 22 gibt die Möglichkeit, über ein einfaches Gesetz die Kündigung eines internationalen Vertrags im Parlament zu beschließen, die allerdings anschließend, genauso wie zuvor der Beitritt, vom Präsidenten ratifiziert werden muss: „Der Antrag an den Präsidenten der Republik Polen zur Kündigung eines mit Zustimmung nach Art. 89, Abs. 1 und Art. 90 der Verfassung der Republik Polen ratifizierten Vertrags erfolgt nach Einholung der in einem Gesetz zum Ausdruck gebrachten Zustimmung [des Parlaments] (Übers. u. Erläut. d. Aut.). Unter Bezugnahme auf Art. 125 der polnischen Verfassung wäre auch die Anordnung eines Austrittsreferendums mit einfacher Mehrheit bei Anwesen- 
heit von mindestens der Hälfte der Abgeordneten durch den Sejm oder über eine Anordnung des Präsidenten bei entsprechender Unterstützung durch den Senat (wiederum einfache Mehrheit bei Anwesenheit der Hälfte der Senatoren) vorstellbar. Aus diesem Grunde hat der im Sommer 2021 in die polnische Politik zurückgekehrte ehemalige Ministerpräsident und EU-Ratsvorsitzende Donald Tusk als eine seiner ersten Initiativen eine bereits in der Vergangenheit von verschiedenen Seiten ins Spiel gebrachte Verfassungsänderung vorgeschlagen, die die Kriterien eines potenziellen EU-Austritts denen des Beitritts (2/3-Mehrheit in beiden Parlamentskammern bei Anwesenheit mindestens der Hälfte der Abgeordneten oder ein Referendum) gleichstellen würde. Trotz einer zunächst nicht völlig ablehnenden Reaktion der PiS sind parlamentarische Schritte bisher ausgeblieben.

\section{Wer hat eigentlich warum ein Interesse an (der Debatte um) einem Polexit?}

Beim genaueren Hinsehen lassen sich drei Gruppen identifizieren, die den Diskurs um einen Polexit aus unterschiedlichen und widersprüchlichen Beweggründen führen:

\section{Die Opposition}

Interessanterweise sind es vor allem die Oppositionsparteien, allen voran Donald Tusk und die von ihm geführte Bürgerplattform (Platforma Obywatelska-PO), die die gegenwärtige Polexit-Debatte befeuern. Zwar ist das Schüren der Befürchtungen, die PiS führe Polen aus der EU, nichts Neues. Sie werden seit Jahren von verschiedenen Seiten geäußert, u. a. mit Blick auf die Justizreformen, die Hetzkampagnen gegen Migranten und LGBTQI-Communities sowie das brüske Auftreten einiger Regierungsvertreter gegenüber strategischen Partnern wie Deutschland oder Frankreich. Nach der Entscheidung des polnischen Verfassungstribunals scheint die stärkste Oppositionsgruppierung nun aber alles auf diese Karte zu setzen - die Mobilisierung der generell mehrheitlich pro-europäisch eingestellten Bevölkerung rund um die Sorge vor dem Verlust von Sicherheit und Wohlstand, die angesichts der gegenwärtig rasant steigenden Inflation und Abgabenlast weiter um sich greifen dürfte. Das politische Kalkül der PO ist also die Rückgewinnung der zuvor wegen deren Wohlfahrtsversprechen an die PiS gegangenen und dort bisher relativ stabil verbliebenen Wählerstimmen. Um diese erneut und nachhaltig auf ihre Seite zu ziehen, müssten eigentlich neue politische Vorhaben etwa im Bereich Sozial- und Wirtschaftspolitik oder Staatsaufbau präsentiert werden. Obwohl derartige Vorschläge (z. B. im Bereich Gesundheitswesen, ökologische Transformation, Dezentralisierung) im Superwahljahr 2019/20 durchaus Teil der politischen Debatte waren, wurden sie bisher nicht weiter konzeptualisiert. Die Opposition reagiert zumeist nur auf kritikwürdige oder verfassungsrechtlich bedenkliche Zustände und verspricht deren Überwindung. Ganz offensichtlich reicht dies aber in keiner Weise aus, um den in den Umfragen seit sieben Jahren bestehenden Vorsprung der Regierung (in der Regel fünf bis zehn Prozent) aufzuholen. Dass die EU-Kommission in der andauernden Auseinandersetzung um die Justizreformen den polnischen Anteil am EU-Wiederaufbaufonds im Rahmen des neuen Rechtsstaatsmechanismus, dessen Rechtmäßigkeit von der PiS-Regierung angefochten wird, bisher nicht ausgezahlt hat, ist in der Lesart der Bürgerplattform eine Bestätigung ihrer Argumentation eines drohenden Polexit. Dadurch werde der faktische Zustand der Negierung europäischer Mindeststandards endgültig offenbart, und da die PiS keinerlei Absicht zu dessen Beseitigung erkennen lasse, sei davon auszugehen, dass sie früher oder später selbst die Initiative zu einem Austritt ergreifen werde. Die PiS-Regierung unterstellt wiederum, Donald Tusk stecke persönlich hinter dieser "erpresserischen « Situation, und sieht ihre These der angeblich antipolnischen Einstellung der "Brüsseler und Berliner Eliten « bestätigt, als deren willfähriger Vollstrecker Tusk vom regierungsnahen Fernsehen gebrandmarkt wird.

Auch Oppositionsparteien der progressiven Linken, die z. B. für die Rücknahme der radikalen Einschränkung des Abtreibungsrechts sowie für dessen grundlegende Liberalisierung eintreten, setzen mitunter auf eine Verbindung der Auseinandersetzung um normativ-politische Postulate mit den rechtlichen, regelbasierten Grundlagen: Die EU wird hier als ein ideelles und wertegeleitetes Gesamtpaket verstanden, in dem strukturelle Aspekte des Funktionierens der Union wie Rechtsstaatlichkeit, Modi zwischenstaatlicher Kooperation etc. mit normativen Aspekten wie sexuellen und reproduktiven Rechten oder Rechten von Minderheiten zusammengedacht werden. Diese Perspektive, der zufolge sich Polen bereits seit Jahren auf dem Pfad eines schleichenden Ausstiegs aus der EU befindet, wird durch eine Reihe von politischen Forderungen aus dem Europaparlament unterstützt (z. B. die "Entschließung vom 21. Oktober 2021 zu der Krise im Zusammenhang mit der Rechtsstaatlichkeit in Polen und dem Vorrang des Unionsrechts«, in der explizit das Urteil des polnischen Verfassungstribunals zur weiteren Einschränkung von Schwangerschaftsabbrüchen vom 22. Oktober 2020 als illegitim und rechtswidrig bezeichnet und als Beispiel für mit Rechtsstaatlichkeit unvereinbares, politisch motiviertes Handeln aufgeführt wird). Mit der Warnung vor einem sukzessiven Polexit wird nicht nur, wie bei der $P O$ und ihren kleineren Partnern im Parteienbündnis Bürgerkoalition (Koalicja Obywatelska - KO), die Hoffnung verbunden, die PiS aus der Regierung zu 
verdrängen, sondern überdies auch die Absicht verfolgt, die inhaltlichen politischen Ziele über die supranationalen Strukturen der EU an den aktuell gegebenen politischen oder gesellschaftlichen Mehrheiten vorbei durchsetzen zu können.

\section{Die Regierungspartei PiS}

Auf der einen Seite weist die PiS den Vorwurf eines angeblich bewussten Kurses auf einen Polexit weit von sich. In seiner Rede vor dem Europaparlament am 19. Oktober 2021 betonte Ministerpräsident Mateusz Morawiecki: "Die europäische Integration stellt für uns eine zivilisatorische und strategische Entscheidung dar. Wir sind in Europa, hier ist unser Platz und das wird sich auch nicht ändern. Wir möchten Europa wieder zu einer starken, ambitionierten mutigen Gemeinschaft machen. Deshalb beschränken wir uns nicht auf kurzfristige Vorteile, sondern sehen auch das, was wir Europa geben können« (siehe »Dokumentation«, Seite 8ff.). Auf der anderen Seite spielt die Regierung aber auch mit dem Polexit-Feuer, und zwar aus drei verschiedenen Gründen:

Erstens aus taktischem Kalkül, kann sie doch eine Furcht vor dem Polexit sowie seinen politischen und vor allem wirtschaftlichen Folgen gegenüber den europäischen Partnern (insbesondere Deutschland) als Druckmittel zur Abwehr von weitreichenden "Strafmaßnahmen" der EU einsetzen. In den Worten von PiSFraktionschef Ryszard Terlecki auf dem Wirtschaftsforum in Karpacz: "Wir sollten darüber nachdenken, wie wir so gut wie möglich zusammenarbeiten können, damit wir alle in der Union sein können, aber in einer Union, die für uns akzeptabel ist - denn wenn es so weiterläuft, wie es derzeit aussieht, dann müssen wir nach drastischen Lösungen suchen [...] Wir wollen nicht austreten [...] aber wir können uns nicht in etwas hineintreiben lassen, das unsere Freiheit und Entwicklung einschränkt. Dies passt zum ideologischen Selbstverständnis der polnischen Nationalkonservativen als europäische Rechtsgaullisten. Als solche betrachten sie die EU als wirksames wirtschafts- und sicherheitspolitisches Instrument zur Entwicklung des Landes, nicht aber als eine weitergehende (Rechts-) Gemeinschaft souveräner Nationalstaaten. Daher verbitten sie sich jegliche "Einmischung« außerhalb von Binnenmarkt und Verteidigungspolitik und spielen in der Hoffnung auf eine ihnen genehmere politische Großwetterlage in Europa auf Zeit. Dem sollten wohl auch die groß inszenierten Gipfeltreffen der politischen Rechten Europas in Warschau (Dezember 2021) und Madrid (Januar 2022) dienen, in denen die PiS europäische Unterstützung für ihre Standhaftigkeit aufzeigt (auch wenn sie sich damit angesichts der konträren Haltung zur Russlandpolitik vieler ihrer potenziellen Koalitionäre, wie etwa Marine Le Pen, auf sehr dünnem Eis bewegt). In einem Inter- view für die Zeitung Polska. The Times vertritt Ryszard Terlecki Mitte Januar dieses Jahres den Standpunkt, bei der Divergenz in der Rechtsstaatsfrage handele es sich um ein in Brüssel, nicht in Warschau, geschaffenes Problem: "Wir werden die Brüsseler Politiker davon überzeugen, dass dies ein Kollisionskurs ist. Wenn sie sich einbilden, dass sie uns auf irgendeine Weise brechen oder uns zu kontroversen Handlungen zwingen können, dann irren sie sich. Dieser Weg führt nirgendwo hin."Letztlich wird also suggeriert, dass ein Polexit am Ende eines nicht aufzulösenden Streits nicht ausgeschlossen werden könne.

Zweitens hat das Anheizen der Polexit-Debatte durch die PiS auch innenpolitische Gründe. Die polnischen Nationalkonservativen haben sich immer wieder als Nationalisten hervorgetan, die in geistiger Verbundenheit mit Carl Schmitt im Zweifel die Politik über das Recht stellen und Demokratie als die Diktatur einer eher beschworenen als realen - Mehrheit verstehen. Die von ihnen auf vielen Feldern erfolgreich betriebene Politik mit der Angst, die sich etwa anhand der Kampagnen gegen LGBTQI-Communities, aber jüngst auch paradigmatisch an der skrupellosen Reaktion auf den durch das Lukaschenko-Regime ausgelösten hybriden Migrationskonflikt an der polnischen EU-Außengrenze zu Belarus (vgl. Polen-Analysen Nr. 286, https://www. laender-analysen.de/polen-analysen/286/) zeigte, könnte langfristig auch den Boden für eine veränderte Einstellung zur EU bereiten. Die Flüchtlingspolitik macht sichtbar, wie manipulierbar, ja sogar umkehrbar die polnische öffentliche Meinung mitunter sein kann. Wie der Soziologe Przemysław Sadura in einer Hintergrundanalyse für die Heinrich-Böll-Stiftung aufgeführt hat, waren bis zum Sommer des Jahres 2015 (und das stabil seit 2004!) ca. drei Viertel der polnischen Bevölkerung für eine Aufnahme von aus politisch und religiösen Gründen verfolgten Menschen; ein Jahr später hingegen sprach sich über die Hälfte von ihnen dagegen aus. Die von der Regierung bewusst herbeigeführte Wagenburgmentalität zur Ablenkung von der humanitären Katastrophe an der belarussischen Grenze hat diese Situation weiter verschärft und die Umgehung verfassungsrechtlicher und internationaler Verpflichtungen, einschließlich der Einschränkung der polnischen Bürgerrechte mit Bezug auf das Grenzgebiet, auf ein bis dato ungekanntes Maß verstärkt - ein Umstand, für den nicht wenige pro-europäisch eingestellte Aktivisten auch die EU verantwortlich machen.

Die Gefahr eines Umkippens der positiven öffentlichen Meinung gegenüber der EU besteht insbesondere dann, wenn die Folgen der konfrontativen Politik der PiS nicht nur wie bisher politische, sondern schmerzhafte ökonomische Kosten verursachen. Es kann nicht davon ausgegangen werden, dass finanzielle Einbußen 
das Konto der Regierung belasten oder die Unterstützung für die Opposition verstärken würden. Aus diesem Grunde hat etwa das Europaparlament gefordert, dass Sanktionen gegen Polen wegen der Missachtung rechtsstaatlicher Prinzipien nicht die Bevölkerung treffen dürften. Da die PiS bereits den Plan verkündet hatte, die nächste Runde ihrer sozialen Wohltaten zum Großteil aus dem EU-Corona-Wiederaufbaufonds zu finanzieren, wird die Umsetzung zielgerichteter Sanktionsmaßnahmen in diesem Zusammenhang komplizierter. Genau vor diesem Hintergrund wird die PiS nicht müde, ihre Auseinandersetzung mit der EU-Kommission in Brüssel, dem Europäischen Gerichtshof in Luxemburg und mittlerweile auch dem Europäischen Gerichtshof für Menschenrechte des Europarats in Straßburg als einen "europäischen Freiheitskampf" gegen die "neokommunistische Bevormundung aus Brüssel und Berlin« darzustellen und dadurch die bisher in überwältigender Mehrheit die EU-Mitgliedschaft unterstützende Bevölkerung sukzessive auf ihre Seite zu ziehen. Schon werden erste Stimmen aus dem Regierungsumfeld laut, die einen Rückzug Polens aus dem Wiederaufbaufonds insgesamt in Erwägung ziehen. Die Chancen der PiS, ihre fortschreitende Selbstisolation auf dem europäischen Parkett für die Konsolidierung ihrer innenpolitischen Macht zu nutzen, stehen besser als manche vermuten oder hoffen dürften. Die Rechtsstaatsmaterie ist enorm komplex, und angesichts des tatsächlich historisch negativen Images des polnischen Justizwesens bleibt bei nicht wenigen polnischen Bürgerinnen und Bürgern dann doch der Eindruck hängen: Was die PiS macht, ist zwar in Teilen fragwürdig, aber wenigstens machen sie irgendetwas - in anderen Bereichen wie etwa der Sozial- und Wirtschaftspolitik haben sie ja schließlich auch geliefert. So überrascht es nicht, dass laut einer Studie des Warschauer Instituts für öffentliche Angelegenheiten (Instytut Spraw Publicznych - ISP) zwar 70 bis 80 Prozent der Oppositionsanhänger der PiS-Regierung die Schuld an der gegenwärtigen Zuspitzung zuschreiben, während bei den Regierungsanhängern über 56 Prozent die Verantwortung in Brüssel und weitere 23 Prozent auf beiden Seiten sehen.

\section{Richtungsstreit innerhalb der Regierung - die Rolle der Solidarna Polska}

Der PiS-Vorsitzende Jaroslaw Kaczyński, Ministerpräsident Morawiecki und Präsident Andrzej Duda streben für Polen einen politischen und der ökonomischen Bedeutung entsprechenden Regionalmachtstatus an. Zudem wird aber deutlich, dass es auch innerhalb des nationalkonservativen Lagers mächtig gärt und wir, drittens, damit Zeugen eines Richtungsstreits sind. Nach dem Rauswurf der vergleichsweise »liberaleren« Koalitionspartei Verständigung (Porozumienie) von Jarosław
Gowin, aber auch unter dem Konkurrenzdruck von Seiten der rechtsradikalen Gruppierung Konföderation (Konfederacja), scheint nunmehr im nationalkonservativen Lager zunehmend die nationalistisch ausgerichtete Kleinpartei Solidarisches Polen (Solidarna Polska) den Ton anzugeben. Unter der Führung des Justizminister-in-Personalunion-Generalstaatsanwalts Zbigniew Ziobro waren deren Vertreter bereits als maßgebliche Triebkräfte hinter der seit 2019 laufenden AntiLGBTQI-Kampagne in Erscheinung getreten. Diese Gruppierung steht für einen kompromisslosen Kurs in der Frage der Justizreformen sowie einen dumpfen Klerikalismus in gesellschaftspolitischen Fragen und hat den Verhandlungsspielraum von Ministerpräsident Morawiecki gegenüber der EU wiederholt massiv zu beschränken gewusst.

Einer ihrer populärsten Vertreter, Ziobros ehemaliger Stellvertreter im Justizministerium und heutiger Europaparlamentarier Patryk Jaki, hat unlängst einen nach ihm benannten Bericht vorgestellt, der die angeblich horrenden Verluste der polnischen Wirtschaft durch die EU-Mitgliedschaft beweisen soll. Eine der Hauptthesen: Die Fördermittel aus den europäischen Fonds seien eine Ausgleichszahlung der EU für den Zugang zum polnischen Markt. Als solche seien sie völlig unzureichend, denn internationale Konzerne würden riesige Summen aus Polen abfließen lassen, aber in jedem Fall könnten sie Polen nicht entzogen werden. Die Aufnahme des Jaki-Berichts in Medien ganz unterschiedlicher politischer Provenienz und gerade unter Wirtschaftsfachleuten ist verheerend. Postwendend erschien zudem ein detaillierter (von der Konrad-Adenauer-Stiftung finanzierter) Gegenbericht der Polnischen Robert-SchumanStiftung (Polska Fundacja im. Roberta Schumana), der die tendenziösen und in Teilen methodologisch unhaltbaren Berechnungen mit eigenen Aufstellungen geradezurücken versuchte. Aber auch wenn hochrangige Vertreter der PiS und des Finanzministeriums sich diese Großerzählung von der Vasallisierung des widerspenstigen Polens in dieser Form nicht zu eigen machen wollen, sollte die Langzeitwirkung dieser Propaganda nicht unterschätzt werden, die - analog zur Brexit-Lüge, welche die britische Bevölkerung vor dem Austrittsreferendum hinsichtlich der Zahlungen Großbritanniens an das EU-Budget in die Irre führte - getrost als Beta-Version einer Polexit-Lüge bezeichnet werden kann.

Der Sejm-Abgeordnete und ehemalige Vizeminister Janusz Kowalski sinnierte schon einmal in den Medien über einen passenden Termin für ein Austrittsreferendum - 2027, zum Ende des laufenden Mehrjährigen Finanzrahmens der EU. Dazu passen das Verhalten der Gewerkschaft Solidarność, die in Luxemburg wegen der Entscheidung des EuGH zum Kohlekraftwerk in Turów gegen die angebliche energiepolitische Knebelung Polens 
protestierte, ebenso wie die enorme staatliche Finanzierung für ultrarechte Gruppierungen, die auch nach polnischem Recht eigentlich vom Verfassungsschutz beobachtet werden müssten. In der Gesamtsicht entsteht der Eindruck, dass hier ein Teil des Regierungslagers bereits an einer politischen Neuausrichtung für die Zeit nach dem Abgang des ohnehin schon zutiefst euroskeptischen Jarosław Kaczyński arbeitet, der in den kommenden fünf Jahren immer wahrscheinlicher wird. Zwar passt ein EU-Austritt ideologisch tatsächlich am ehesten zur Partei Solidarisches Polen, aber auch bei dieser Gruppierung sollte eher strategisches Kalkül vermutet werden. Ihr geht es um die Kreierung einer politischen Welle, auf der sie an die Macht surfen können, egal, ob (finanziell notgedrungen) weiterhin innerhalb oder (ideologisch gesehen lieber) außerhalb der EU.

\section{Auf dem Weg in den EWR?}

Aber es gibt durchaus auch ökonomische Bedingungen, die in Zukunft weitere Argumente für die entfachte Polexit-Debatte bieten könnten. Die polnische Wirtschaft hat dank ihrer Teilnahme am EU-Binnenmarkt einen enormen Aufstieg vollzogen. Zwar liegt Polen mit Blick auf das Bruttoinlandsprodukt pro Kopf noch deutlich hinter seinem Nachbarn Tschechien, aber Portugal wurde bereits eingeholt, und das Aufschließen zu Ländern wie Spanien oder Italien liegt im Laufe dieses Jahrzehnts durchaus im Bereich des Möglichen (siehe Grafik 1 auf S. 12). Dem Brexit vergleichbare oder ihn gar übertreffende Verwerfungen dürften daher keinesfalls im Interesse der polnischen Wirtschaftskapitäne und Staatenlenkenden sein. Andererseits ist da das ewige Thema der von Polen aus makroökonomischen (Abwertungsspielräume), identitätspolitischen (Symbol der Staatlichkeit) und psychologischen (gefühlt geschrumpftes Portemonnaie) Gründen auf den Sankt Nimmerleinstag verschobenen Euro-Einführung sowie die Prognose, dass Polen dank seiner Wirtschaftsleistung in nicht allzu ferner Zukunft zum EU-Nettozahler werden dürfte. Interessanterweise mag dies für einen Teil der Bevölkerung nicht etwa ein Grund von Stolz darstellen, sondern durchaus Anlass zu kühlen Kalkulationen bieten.

Wenn nämlich die europäischen Fonds, wie der Bericht der Polnischen Robert-Schuman-Stiftung unterstreicht, tatsächlich lediglich eine Unterstützungsfunktion bei der wirtschaftlichen Entwicklung haben und die Auswirkungen der Teilnahme am europäischen Binnenmarkt um ein Dreifaches höher anzusetzen sind, dann könnte sich für bestimmte Kräfte auf der politischen Rechten durchaus die Frage stellen, inwiefern nach Ende der Fördermittelzahlungen eigentlich noch »substanziell" von der EU-Mitgliedschaft zu profitieren wäre, wenn man ohnehin geschätztes Mitglied der NATO ist. Vielleicht schwebt einigen "Polexitologen« gar ein Polen als ultrakonservatives Pendant der Schweiz oder Norwegens vor. Also eines wirtschaftlich für globale Lieferketten und Produktionsprozesse wichtigen Landes mit direktem Zugang zum europäischen Binnenmarkt, welches sich von jeglicher »Brüsseler Einmischung in seine inneren Angelegenheiten « bzw. einem wachsenden »Rechtsprechungsimperialismus» (Prof. jur. Dariusz Dudek, Berater von Präsident Andrzej Duda) befreit hat. Prof. Zbigniew Krysiak, einer der beiden Autoren des Jaki-Berichts, beschrieb die Lage nach dem Urteil des polnischen Verfassungstribunals im klerikalen Sender Radio Maryja folgendermaßen: „Es wird ein Krieg geführt, in dem wir nicht aufgeben dürfen. Das Schlimmste sind die Folgen des Verlusts des christlichen Geistes und des Freiheitsgefühls.«

\section{Die Gefahr eines Polexit: viel Lärm um nichts?}

Der Blick auf die Gemütslage der polnischen Gesellschaft ist also ambivalent. Die Zustimmungswerte für die EU-Mitgliedschaft sind weiterhin an der europäischen Spitze. Allerdings dürfen allgemeine Bekenntnisse zur EU-Mitgliedschaft keineswegs mit einer Zustimmung zu allen zentralen Politikbereichen oder zum Funktionieren der Europäischen Union als solcher gleichgesetzt werden. So ist in einer aktuellen repräsentativen Umfrage zur Zusammenarbeit Polens, Deutschlands und Frankreichs im Weimarer Dreieck (siehe Grafik12 auf Seite 18) zu lesen, dass bereits heute in allen drei Ländern relative Mehrheiten für eine Rücknahme von bestimmten an die EU übergebenen Souveränitätsrechten zugunsten ihrer Länder sind: 38 Prozent der befragten Polen, 33 Prozent der Franzosen und sogar 46 Prozent der Deutschen (36 Prozent aus Polen, 28 Prozent aus Frankreich und 29 Prozent aus Deutschland sprachen sich für einen weiteren Souveränitätsgewinn der EU aus). Zwar würde, wie die Studie des Instituts für Öffentliche Angelegenheiten aufzeigt, eine große Mehrheit (79 Prozent) der polnischen Bevölkerung heute noch gegen einen Polexit stimmen (siehe Grafik 11 auf S. 17), aber deutlich wird in den Umfragen auch: Die Botschaft von der kulturell-ideologischen "Diktatur« der EU kommt gerade bei konservativen Kreisen gut an.

Das Neue an der gegenwärtigen Debatte um einen Polexit ist, dass sich bisher sogar in durchaus EU-kritischen Kreisen nie wirklich ein tatsächliches Austrittsszenario wahrnehmen ließ, sondern stets nur von der erwähnten Umstrukturierung der EU zurück zu mehr nationalstaatlicher Souveränität die Rede war. Die Verwendung des Begriffs Polexit suggeriert also eine gewisse neue Dringlichkeit. Überdies besteht natürlich die Gefahr, dass sich bestimmte Akteure bei der PiS mit ihrer unrealistischen Argumentation in eine Sackgasse manövrieren, aus der sie ohne Gesichtsverlust nicht 
mehr herausfinden können. So könnte wie beim Brexit ein gewisser Automatismus entstehen, der zum Stellen der Gretchenfrage führt. In jedem Fall ist bei einer weiteren Eskalation des Rechtsstaatskonflikts davon auszugehen, dass sich in Polen mittelfristig ein Wählerpotenzial von EU-Unzufriedenen aufbaut, das von radikaleren Strömungen angezapft werden kann.

Der Streit um die Rechtsstaatlichkeit zwischen Polen und der EU wird sich aller Voraussicht nach schon bald weiter zuspitzen. Polen und Ungarn haben die Rechtmäßigkeit des neuen Rechtsstaatsmechanismus angefochten, das EuGH-Urteil wird nun für Mitte Februar erwartet. Das polnische Verfassungstribunal wird sich zur gleichen Zeit auf Antrag von Justizminister Ziobro mit der Verfassungsmäßigkeit eben jenes Instruments befassen. Wie das Tribunal entscheiden wird, dürfte auf der Hand liegen (zumal der Berichterstatter in diesem Fall Stanisław Piotrowicz ist, der noch als Sejm-Abgeordneter bis 2019 einer der Hardliner hinter der Justizreformen war und anschließend mit einer Position als Verfassungsrichter »belohnt« wurde, obwohl er bereits die gesetzliche Altersgrenze von 67 Jahren überschritten hatte). Ob die PiS-Regierung also vor den nächsten regulären Wahlen im Herbst 2023 doch noch zu substanziellen Zugeständnissen im Sinne einer Auflösung der Kontroverse bereit sein könnte, erscheint derzeit wenig wahrscheinlich - auch wenn Präsident Duda jüngst in Brüssel bei EU-Kommissionspräsidentin Ursula von der Leyen und dem Präsidenten des Europäischen Rates Charles Michel einen Kompromissvorschlag unterbreitet hat: Seine gesetzgeberische Initiative, die eine Überführung der vom EuGH inkriminierten Disziplinarkammer am Obersten Gerichtshof in eine neue Kammer für berufliche Verantwortung (von Juristen) vorschlägt, wird bisher weder von der Regierung mitgetragen noch sehen Opposition und unabhängige Juristen darin eine gangbare Lösung. Beim genaueren Hinsehen blieben auch hier Zweifel, ob diese Maßnahme nicht wiederum die Unabhängigkeit der Richterschaft untergrabe.

Vor diesem Hintergrund kann nicht ausgeschlossen werden, dass - genügend Renitenz von Seiten der $P i S$ vorausgesetzt - entscheidende politische Akteure in der EU irgendwann genug vom erwartbaren Pingpong haben. Zumal dann, wenn »der polnische Weg« weiter Schule machen sollte, denn neben Ungarn liegt nun auch Rumänien in Fragen der Rechtsstaatlichkeit und des Vorrangs von EU-Recht im Streit mit dem EuGH. Dann könnte eine Situation entstehen, in der EU-Mitgliedsstaaten ein innen- oder außenpolitisches Interesse daran haben könnten, Polens Politik als inakzeptabel und unvereinbar mit EU-Kriterien zu deklarieren und es damit trotz der erwartbar verheerenden Auswirkungen für den europäischen Zusammenhalt und das Funktionieren des europäischen Binnenmarkts herauszutreiben, um damit ein unmissverständliches Signal auszusenden. Der Verlust Polens könnte für die Anhänger einer stärkeren politischen Integration der EU in Richtung eines föderalen Bundesstaats weniger schwer wiegen als ein Dauerkonflikt mit einem auf seine nationale Souveränität fixierten Mitgliedsstaat.

Wie die Antrittsbesuche zunächst von Bundesaußenministerin Annalena Baerbock und dann von Bundeskanzler Olaf Scholz in Warschau zeigten, steht die neue Bundesregierung hier vor einer schwierigen Aufgabe. Nicht nur, weil der Koalitionsvertrag der neuen AmpelRegierung in Berlin den Kurs auf vertiefte EU-Integration setzt, was von Jarosław Kaczyński bereits mit dem "Aufbau eines Vierten Reiches" gleichgesetzt wurde. Sie sollte sich einerseits nicht auf den Polexit-Alarmismus einlassen und der PiS nicht das Gefühl geben, Deutschland wolle einen Austritt Polens unter allen Umständen vermeiden, um sich nicht erpressbar zu machen. Gleichzeitig darf die PiS mit ihrer Unterhöhlung des Rechtsstaats nicht durchkommen. Berlin wäre gut beraten so wie jüngst von Reinhard Bütikofer (Bündnis 90/Die Grünen), Michael Link (FDP) und Dietmar Nietan (SPD) in einem gemeinsamen Beitrag für den Tagesspiegel gefordert - endlich substanziell auf bilaterale und europäische Anliegen Polens, wie etwa bei der Politik gegenüber der Ukraine sowie Russland, der Energie- und Sicherheitspolitik, Nord Stream 2, der Entsenderichtlinie, Wiedergutmachung etc., einzugehen. Darauf zu warten, dass die PiS eines Tages die Macht wird abgeben müssen, ist weder eine politisch tragfähige Option noch liegt das im deutschen Interesse - schon deshalb, weil nicht mit Sicherheit davon auszugehen ist, dass das Pendel wieder mehr in Richtung politische Mitte und nicht gar weiter nach rechts ausschlagen wird.

Deutschland, das mit Polen seit 30 Jahren über einen Nachbarschaftsvertrag und tiefgreifende wirtschaftliche wie kulturelle Verflechtungen verbunden ist sowie einige Millionen Bürger mit polnischer Abstammung in seiner Bevölkerung aufzuweisen hat, könnte so der polnischen Öffentlichkeit signalisieren, dass die PiS-Propaganda von der angeblich antipolnischen Haltung Berlins und Brüssels jeglicher Grundlage entbehrt, sondern berechtigte nationale Interessen auf faire Weise im Rahmen der EU und ihrer Institutionen verhandelt werden können.

Eine frühere Version der Analyse erschien unter "Wer spielt hier warum mit dem Feuer? Zum Wirklichkeitsgehalt der Gefahr eines Polexits", Zentrum Liberale Moderne, 6. Dezember 2021 https://libmod.de/ spiel-mit-feuer-gefahr-polexit-hahn-fuhr-roehrborn/ 


\section{Über die Autoren}

Irene Hahn-Fuhr ist Mitglied der Geschäftsführung beim Zentrum Liberale Moderne, Berlin. Nach Stationen u. a. als Büroleiterin beim europapolitischen Sprecher der Fraktion Bündnis90/Die Grünen im Deutschen Bundestag und bei der Deutschen Gesellschaft für Auswärtige Politik (DGAP) leitete sie zuletzt das Auslandsbüro der Heinrich Böll Stiftung in Warschau. Die Politologin ist u. a. Mitglied im Stiftungsrat der Stiftung Kreisau für Europäische Verständigung und der Kopernikus-Gruppe.

Gert Röhrborn, Politologe, lebt als freiberuflicher Autor, Trainer und Übersetzer in Warschau und Belgrad. Er war in den vergangenen fünfzehn Jahren bei der Durchführung großer europäischer Projekte u. a. an der TU Dresden, als Büroleiter des Berliner Netzwerks Citizens of Europe und bei der Polnischen Robert-Schuman-Stiftung (Polska Fundacja im. Roberta Schumana) in Warschau tätig. In den Jahren 2014 bis 2021 leitete er das Programm für »Demokratie und Menschenrechte« im Warschauer Büro der Heinrich-Böll-Stiftung.

\section{DOKUMENTATION}

\section{Rede von Ministerpräsident Mateusz Morawiecki vor dem Europäischen Parlament}

\section{[Auszüge]}

19. Oktober 2021

\section{Sehr geehrter Herr Vorsitzender,} sehr geehrte Frau Vorsitzende, meine Damen und Herren Abgeordnete,

ich stehe heute vor Ihnen im Parlament, um unseren Standpunkt zu einigen grundlegenden Fragen darzulegen, die ich für die Zukunft der Europäischen Union für entscheidend halte. Nicht nur für die Zukunft Polens, sondern auch für die Zukunft unserer gesamten Union.

Erstens möchte ich auf die Krisen eingehen, mit denen Europa aktuell konfrontiert ist und mit denen wir uns befassen müssen.

Zweitens werde ich über Standards und Regeln sprechen, die immer und für alle gleich sein müssen - und darüber, dass dies zu oft nicht der Fall ist.

Drittens werde ich unsere Sicht auf die Grundsätze darlegen, laut derer kein öffentliches Verwaltungsorgan Maßnahmen tätigen darf, für die keine Rechtsgrundlage existiert.

Der vierte Punkt meiner Rede wird das Urteil des polnischen Verfassungsgerichts betreffen - und die Frage, was dieses und andere ähnliche Urteile für die EU bedeuten. Und ich werde betonen, wie wichtig Vielfalt und gegenseitiger Respekt sind.

Im fünften Punkt werde ich unseren Standpunkt zum verfassungsrechtlichen Pluralismus erläutern.

Und anschließend möchte ich die gewaltigen Risiken für die gesamte Gesellschaft thematisieren, die sich aus der Anwendung des Urteils des Gerichtshofs der Europäischen Union ergeben und die sich in Polen bereits materialisieren.

Zum Schluss werde ich alle Schlussfolgerungen zusammenfassen und einen, wie ich glaube, hoffnungsvollen Blick in die Zukunft wagen.

$[\ldots]$

Nun möchte ich einige Worte über den Beitrag Polens zu unserem gemeinsamen Projekt sagen.

Die europäische Integration stellt für uns eine zivilisatorische und strategische Entscheidung dar. Wir sind in Europa, hier ist unser Platz und das wird sich auch nicht ändern. Wir möchten Europa wieder zu einer starken, ambitionierten mutigen Gemeinschaft machen. Deshalb beschränken wir uns nicht auf kurzfristige Vorteile, sondern sehen auch das, was wir Europa geben können.

Polen profitiert von der Integration vor allem durch den Handel im Rahmen des gemeinsamen Binnenmarktes. Technologietransfers und Direkttransfers spielen ebenfalls eine wichtige Rolle. Aber Polen ist der Union nicht mit lee- 
ren Händen beigetreten. Die wirtschaftliche Integration Polens erweiterte die Möglichkeiten für Firmen aus meinem Land, eröffnete aber auch deutschen, französischen oder niederländischen Unternehmen riesige Chancen. Unternehmer aus diesen Ländern profitieren in großem Umfang von der EU-Erweiterung.

Hier genügt es, den gewaltigen Abfluss von Dividenden, Zinseinnahmen und Gewinnen aus anderen Finanzinstrumenten aus den weniger wohlhabenden mitteleuropäischen Ländern in die reicheren Länder in Westeuropa zu betrachten. Wir hingegen wollen, dass es bei dieser Zusammenarbeit keine Verlierer gibt, sondern ausschließlich Gewinner.

Polen hat sich für einen ehrgeizigen Aufbaufonds stark gemacht, um die heutige Antwort auf die Herausforderungen der Transformation in den Bereichen Klima, Energie und Pandemiefolgen an die realen Bedürfnisse anzupassen. Um ein starkes Wirtschaftswachstum zu ermöglichen, das Millionen von Kindern, Frauen und Männern Mut macht und sie der Globalisierung nicht hilflos ausliefert. In diesen Fragen stimmten wir mit dem Europäischen Parlament voll und ganz überein.

Polen unterstützt den europäischen Binnenmarkt tatkräftig. Wir wollen eine strategische Autonomie, die unsere 27 Länder stärkt.

Deshalb setzen sich Polen, Deutschland, Tschechien und andere mitteleuropäische Staaten für Lösungen ein, die die Wettbewerbsfähigkeit der europäischen Wirtschaft im Geiste der Verwirklichung der vier Grundfreiheiten stärken: Warenverkehrsfreiheit, Dienstleistungsfreiheit, Personenverkehrsfreiheit und Kapitalverkehrsfreiheit. Und zwar ohne die Unterstützung sogenannter Steuerparadiese, wie dies noch immer seitens einiger westeuropäischer Länder geschieht, und zwar zum Nachteil ihrer Nachbarn. Ja, meine verehrten Damen und Herren Abgeordnete: Steuerparadiese, die wir in der EU dulden, führen zur Aneignung von Geldern durch die Reichsten. Ist dies gerecht? Hilft dies, das Schicksal der Mittelklasse oder der ärmeren Schichten zu verbessern? Ist dies konform mit dem europäischen Wertekatalog? Ich habe große Zweifel.

Polen setzt sich zusammen mit dem Rest Mitteleuropas auch für eine ehrgeizige EU-Erweiterungspolitik ein, die Europa in der Westbalkanregion stärkt. Dies verstehen wir als geografische, historische und strategische Ergänzung der europäischen Integration. Wir sagen ja zu globalen Ambitionen der Union und sprechen uns für eine starke europäische Verteidigungspolitik aus, mit einer Struktur, die mit der NATO vollumfänglich kohärent ist.

Aktuell sind wir Zeugen eines organisierten Angriffs an der Ostgrenze der Union, der die Migration aus dem Nahen Osten zynisch zur Destabilisierung missbraucht. Und heute ist Polen der Sicherheitsgarant der EU, indem wir gemeinsam mit Litauen und Lettland einen Schutzschild zur Verteidigung der Grenze bilden. Und durch die Stärkung unseres Verteidigungspotenzials verbessern wir auch die Sicherheit der Union, im traditionellsten Sinne dieses Worts. [...]

Während heute in den Gründungsstaaten der Gemeinschaft das Vertrauen zur Union auf einen historischen Tiefpunkt gesunken ist (z.B. 36\% in Frankreich), bleibt dieser Wert in Polen auf einem Höchststand. Über 85\% der polnischen Bürger sagen klar: Polen ist EU-Mitglied und wird dies auch bleiben. Meine Regierung und die parlamentarische Mehrheit, die hinter uns steht, ist Teil dieser proeuropäischen Mehrheit in Polen.

Dies bedeutet nicht, dass in Polen keine Zweifel und Sorgen bezüglich der Änderungstrends in Europa existieren. Diese Sorgen gibt es, und sie sind leider auch begründet.

Ich habe eben davon gesprochen, wie viel Polen in die EU eingebracht hat. Aber leider hören wir noch immer von einer Einteilung in bessere und schlechtere Länder. Zu oft haben wir es mit einem Europa der doppelten Standards zu tun. Und jetzt möchte ich erläutern, warum wir mit diesem Modell abschließen müssen.

Heute erwarten alle Europäer nur eines von uns. Die Menschen wollen, dass wir den Herausforderungen die Stirn bieten, die für die aktuell kumulierten Krisen verantwortlich sind. Gemeinsam und nicht gegeneinander, ohne die zwanghafte Suche nach Schuldigen - oder eher jenen, die in Wirklichkeit zwar keine Schuld tragen, sich aber bequem beschuldigen lassen.

Leider stellen sich viele Bürger unseres Kontinents angesichts einiger Praktiken in den EU-Institutionen heute die Frage, ob vor dem Hintergrund extrem unterschiedlicher Urteile und Entscheidungen aus Brüssel und Luxembourg gegenüber verschiedenen Mitgliedsstaaten, die unter vergleichbaren Umständen fallen und damit die Gegensätze zwischen dem starken, alten und neuen Europa, starken und schwachen Ländern, reichen und weniger reichen Staaten de facto vertiefen, tatsächlich von Gleichberechtigung gesprochen werden kann.

[...]

Die Spielregeln müssen für alle gleich sein. Zu ihrer Einhaltung sind wir alle verpflichtet - auch die Institutionen, die kraft dieser Verträge ins Leben gerufen wurden. Darauf beruht Rechtsstaatlichkeit.

Die Ausweitung von Kompetenzen oder die Methode der vollendeten Tatsachen ist unzulässig. Unzulässig ist auch, anderen eigene Entscheidungen ohne Rechtsgrundlage aufzuzwingen. Und umso mehr ist es unzulässig, zu diesem Zweck finanzielle Erpressung anzudrohen, Strafen ins Spiel zu bringen oder noch weitergehende Worte gegenüber einigen Mitgliedsstaaten zu verwenden. 
Ich lehne eine Sprache ab, in der Drohungen, Warnungen und Erpressungen dominieren. Und ich bin nicht damit einverstanden, dass Politiker Polen erpressen oder einschüchtern. Dass Erpressungen zu einer politischen Methode gegenüber anderen EU-Mitgliedsstaaten werden. So funktioniert Demokratie nicht.

[...]

Sehr verehrte Abgeordnete. Nun einige Worte zum Thema Rechtsstaatlichkeit.

$[\ldots]$

EU-Recht hat Vorrang vor nationalem Recht - bis zur Gesetzesebene und in den Bereichen, in denen der EU entsprechende Kompetenzen übertragen wurden. Dieser Grundsatz gilt in allen EU-Ländern. Die Verfassung aber bleibt das ranghöchste Recht.

Wenn die kraft der EU-Verträge berufenen Institutionen ihre Kompetenzen überschreiten, müssen die Mitgliedsstaaten Instrumente haben, um reagieren zu können.

Die Union ist eine große Errungenschaft der Länder Europas. Sie ist ein starker wirtschaftlicher, politischer und gesellschaftlicher Bund. Sie ist die stärkste und am besten entwickelte internationale Organisation in der Geschichte. Aber die Europäische Union ist kein Staat. Staaten sind dagegen die 27 Mitgliedsländer der Union! Und diese Staaten bleiben der europäische Souverän - als »Herren der Verträge». Und die Staaten sind es auch, die über den Umfang der an die Europäische Union übertragenen Kompetenzen entscheiden.

In den Verträgen haben wir der Union sehr weitreichende Kompetenzen verliehen. Aber wir haben ihr nicht alle Aufgaben übertragen. Viele Rechtsbereiche bleiben in der Kompetenz der Nationalstaaten.

Wir haben keine Zweifel bezüglich des Vorrangs des europäischen Rechts vor nationalen Gesetzen in allen Bereichen, in denen die Mitgliedsstaaten der Union entsprechende Kompetenzen übertragen haben.

Allerdings stellt das polnische Verfassungsgericht ähnlich wie Gerichtshöfe in vielen anderen Ländern die Frage, ob das Monopol des Europäischen Gerichtshofs zur Festlegung der tatsächlichen Grenzen dieser Kompetenzübertragung die richtige Lösung ist. Da dieser Bereich das Verfassungsrecht berührt, muss es jemanden geben, der die Verfassungsgemäßheit derartiger eventueller neuer Kompetenzen überprüft, insbesondere in einer Situation, in der der Europäische Gerichtshof immer weitreichendere Kompetenzen der EU-Institutionen aus den Verträgen ableitet.

Ansonsten wäre es sinnlos gewesen, Artikel 4 in den Vertrag über die Europäische Union aufzunehmen, der von der Anerkennung der politischen und verfassungsrechtlichen Strukturen der Mitgliedsstaaten durch die Union spricht. Es wäre auch sinnlos gewesen, Artikel 5 in den Vertrag aufzunehmen, laut dem die Union nur im Rahmen der ihr übertragenen und zuerkannten Kompetenzen tätig werden darf. Beide Artikel wären sinnesleer, ja, sinnesleer!, wenn außer dem Europäischen Gerichtshof niemand das Recht hätte, hinsichtlich der verfassungsrechtlichen Bedingungen der nationalen Rechtsordnung das Wort zu ergreifen.

Mir ist bewusst, dass das kürzlich ergangene Urteil des polnischen Verfassungsgerichts Gegenstand eines grundlegenden Missverständnisses geworden ist. Wenn ich hören würde, dass das Verfassungsgericht eines anderen Landes die EU-Verträge für ungültig erklärt, wäre ich ebenfalls verwundert. Aber vor allem würde ich versuchen in Erfahrung zu bringen, was genau im Urteil des Gerichts steht.

[...]

Der Begriff verfassungsrechtlicher Pluralismus bedeutet, dass es zwischen uns und unseren Justizsystemen Raum für Dialog gibt. Dieser Dialog erfolgt auch mittels Richterentscheidungen. Wie sonst sollten sich Gerichte verständigen, wenn nicht durch ihre Entscheidungen? Es darf jedoch keine Zustimmung zur Erteilung von Anweisungen und Befehlen an einzelne Länder geben. Das ist nicht das Wesen der Europäischen Union.

Wir haben vieles gemeinsam und möchten noch mehr gemeinsam haben - aber es gibt auch Unterschiede zwischen uns. Wenn wir zusammenarbeiten wollen, müssen wir uns mit der Existenz dieser Unterschiede abfinden, sie akzeptieren und einander respektieren.

Die Union wird nicht deshalb zerfallen, weil unsere Rechtssysteme unterschiedlich sind. Wir funktionieren bereits seit 70 Jahren auf diese Weise. Vielleicht werden wir in Zukunft Änderungen vornehmen, die unsere Gesetzgebung noch weiter angleichen. Aber dafür sind Entscheidungen der souveränen Mitgliedsstaaten erforderlich.

Heute können wir zwischen zwei Standpunkten wählen: entweder nehmen wir alle über das Recht und die Verträge hinausgehenden Versuche zur Beschränkung der Souveränität der Staaten Europas (darunter auch Polens) hin und lassen die schleichende Kompetenzerweiterung von Organen wie dem Europäischen Gerichtshof gewähren, d.h. eine "stille Revolution", die nicht auf Grundlage demokratischer Entscheidungen, sondern mit Hilfe von Gerichtsurteilen erfolgt - oder wir sagen: „Nein, meine Lieben«! Wenn Ihr aus Europa einen supranationalen Superstaat machen wollt, dann holt gefälligst zuerst die Zustimmung aller Länder und Gesellschaften Europas ein. 
Ich wiederhole es noch einmal: das oberste Recht der Republik Polen ist die Verfassung. Sie hat Vorrang vor anderen Rechtsquellen. Von diesem Grundsatz kann kein polnisches Gericht, kein polnisches Parlament und keine polnische Regierung abweichen.

An dieser Stelle ist aber auch zu betonen, dass das polnische Verfassungsgericht nie festgestellt hat - auch nicht im kürzlich ergangenen Urteil - dass die Vorschriften des Vertrags über die Europäische Union als Ganzes gegen die polnische Verfassung verstoßen. Ganz im Gegenteil! Polen hält sich vollumfänglich an die Verträge.

Und genau deshalb hat das polnische Verfassungsgericht geurteilt, dass eine sehr konkrete Auslegung einiger Vorschriften des Vertrags verfassungswidrig ist, die sich aus der neuesten Rechtsprechung des Europäischen Gerichtshofs ergibt.

[...]

Mit Sicherheit lohnt sich eine Debatte darüber, ob die Union verändert werden sollte. Sollte der EU-Haushalt nicht größer ausfallen? Sollen wir nicht mehr in die gemeinsame Sicherheit investieren? Sollten die Verteidigungsausgaben nicht von den Haushaltsdefizitmechanismen ausgenommen werden? Polen schlägt eben dies vor! Sollten wir unsere Fähigkeit zur Abwehr von hybriden Gefahren und Cyber-Attacken nicht verbessern? Sollen wir Investitionen in strategische Wirtschaftsbranchen nicht besser kontrollieren? Wie lässt sich die Energie- und Klimatransformation gerecht und effektiv finanzieren? Wie kann unser Entscheidungsprozess nicht effektiver werden? Was tun, damit sich unsere Bürger in der Union nicht immer stärker entfremdet fühlen?

Ich stelle diese Fragen, weil ich überzeugt bin, dass die Antworten über die Zukunft der Union entscheiden.

Über all dies sollten wir debattieren. [...]

Verehrtes Parlament! Ich will ein starkes und großes Europa. Ein Europa, das um Gerechtigkeit, Solidarität und Chancengleichheit kämpft. Ein Europa, das fähig ist, autoritären Regimen die Stirn zu bieten. Ein Europa, das auf die neusten wirtschaftlichen Rezepte setzt. Ein Europa, dass die Kultur und Tradition zu schätzen weiß, aus der es entstanden ist. Ein Europa, das die Herausforderungen der Zukunft erkennt und an den besten Lösungen für die gesamte Welt arbeitet.

Das ist eine große Aufgabe für uns. Für uns alle, liebe Freunde. Nur so finden die Bürger die Hoffnung auf eine bessere Welt von morgen wieder. Nur so finden sie den Willen wieder, aktiv zu werden und zu kämpfen. Dies ist eine schwierige Aufgabe. Aber lassen Sie sie uns angehen. Lassen Sie sie uns gemeinsam angehen. Lang lebe Polen, lang lebe eine Europäische Union der souveränen Nationalstaaten, lang lebe Europa, der großartigste Ort der Welt!

Vielen Dank.

Quelle: https://www.gov.pl/web/primeminister/rede-von-premierminister-mateusz-morawiecki-vor-dem-europischenparlament (deutsche Übersetzung; abgerufen am 10.02.2022). 


\section{Veränderungen des Bruttoinlandsprodukts Polens pro Kopf seit dem Beitritt Polens zur Europäischen Union}

Grafik 1: Veränderungen des Bruttoinlandsprodukts Polens pro Kopf seit dem Beitritt Polens zur Europäischen Union

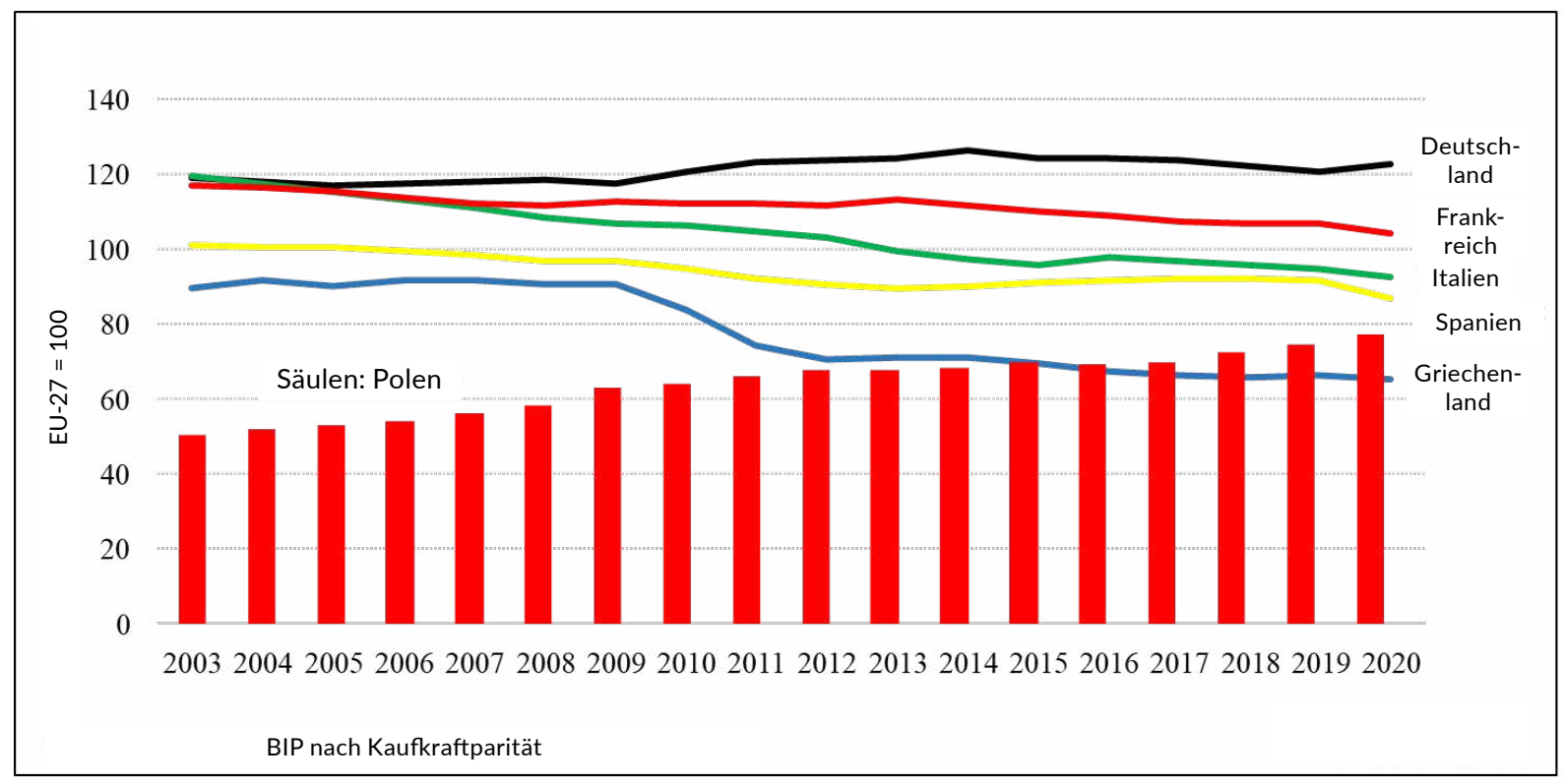

Quelle: Duszczyk, Maciej; Götz, Marta; Gorzelak, Grzegorz u. a.: Gdzie naprawdę sq konfitury? Najważniejsze gospodarcze korzyści członkostwa Polski w Unii Europejeskiej [Wo sind denn wirklich die Konfitüren? Die wichtigsten wirtschaftlichen Vorteile der Mitgliedschaft Polens in der Europäischen Union]. Warszawa: Polska Fundacja im. Roberta Schumana 2021, https://schuman.pl/publikacje/; Quelle der Daten: Eurostat.

\section{Einstellungen zur Europäischen Union}

Grafik 1: Unterstützen Sie die Mitgliedschaft Polens in der Europäischen Union? (\%, Okt. 2021)

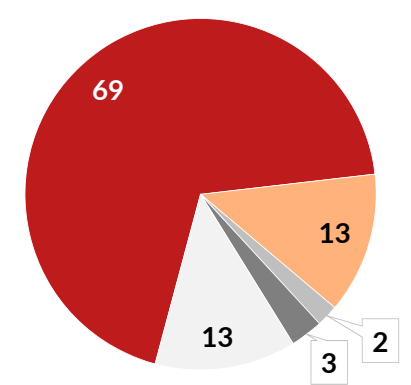

- Ich unterstütze sie eindeutig.

- Ich unterstütze sie.

- Ich bin dagegen.

- Ich bin eindeutig dagegen.

Ich habe keine Meinung dazu.

Quelle: Kucharczyk, Jacek: Widmo Polexitu? Społeczne postawy wobec członkostwa Polski w Unii Europejskiej [Das Gespenst des Polexit? Einstellungen der Gesellschaft gegenüber der Mitgliedschaft Polens in der Europäischen Union]. Warszawa: Instytut Spraw Publicznych (ISP) 2021. https://www.isp. org.pl/ (abgerufen am 01.02.2022). 
Grafik 2: Bringt Ihrer Meinung nach die Mitgliedschaft in der Europäischen Union Polen mehr Vorteile oder mehr Nachteile? (\%, Okt. 2021)

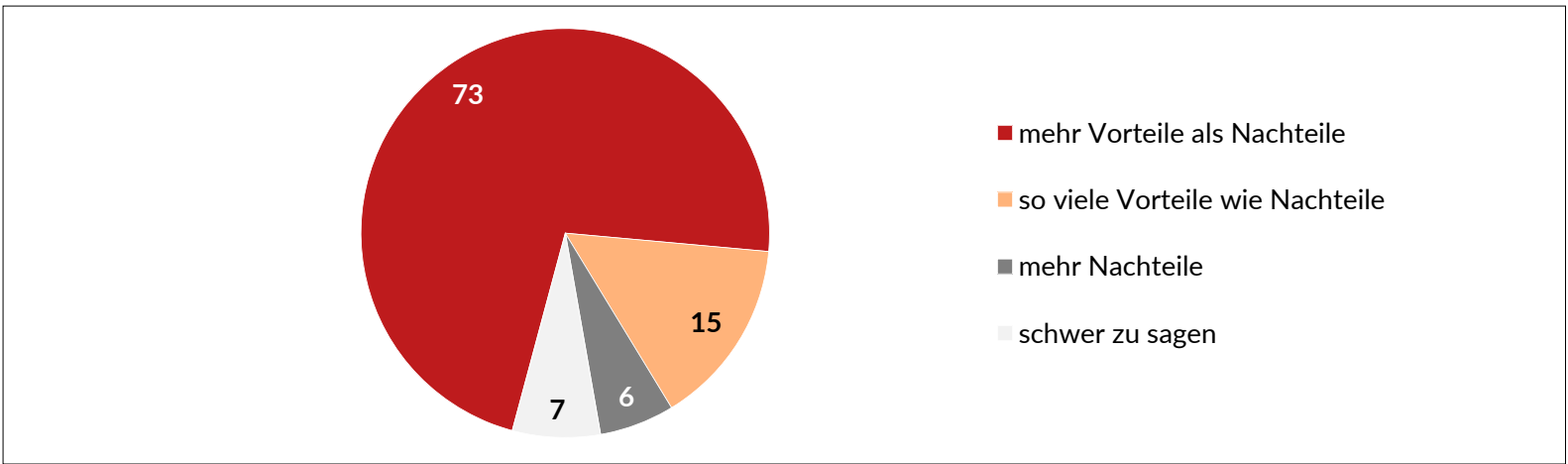

Quelle: Kucharczyk, Jacek: Widmo Polexitu? Społeczne postawy wobec członkostwa Polski w Unii Europejskiej [Das Gespenst des Polexit? Einstellungen der Gesellschaft gegenüber der Mitgliedschaft Polens in der Europäischen Union]. Warszawa: Instytut Spraw Publicznych (ISP) 2021. https://www.isp. org.pl/ (abgerufen am 01.02.2022)

\section{Grafik 3: Wie sehr stimmen Sie den folgenden Aussagen zu? (positive Antworten, \%, Okt. 2021)}

Ich stimme zu. I Ich stimme eindeutig zu.

$\begin{array}{llllllll}0 & 10 & 20 & 30 & 40 & 50 & 60 & 70\end{array}$

Die EU-Institutionen behandeln Rechtsverletzungen oder Verletzungen von EU-Prinzipien vonseiten verschiedener EU-Länder nicht immer gleich.

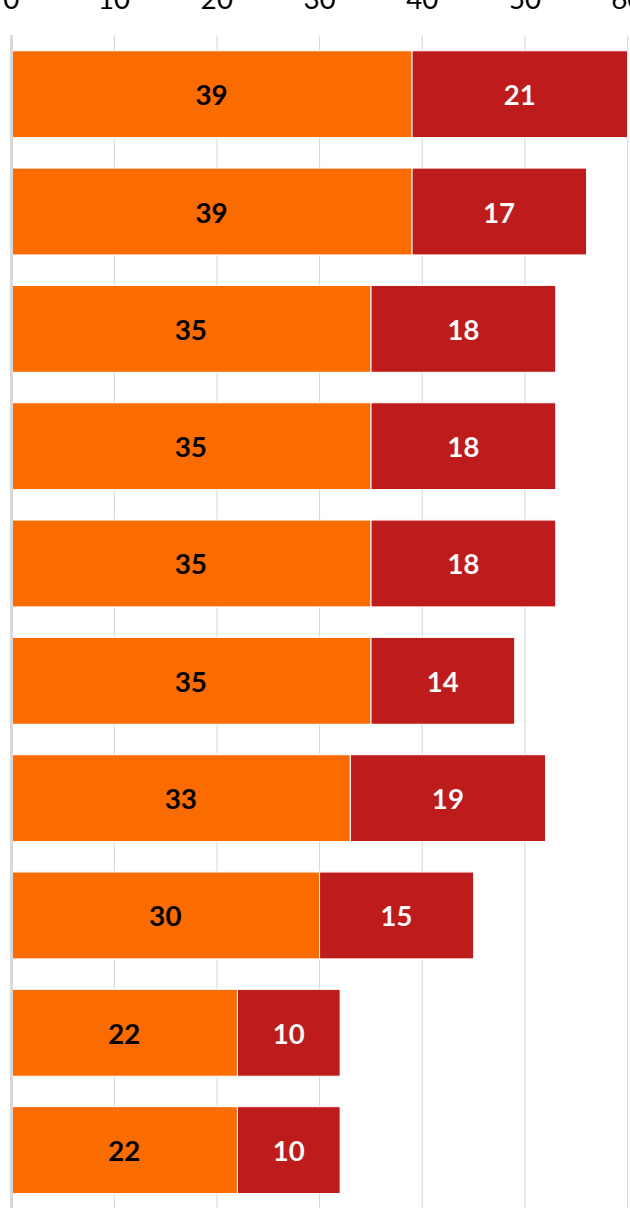

Große ausländische Unternehmen aus anderen EU Ländern treten in den polnischen Markt ein und verdrängen polnische Unternehmen.

Die EU will Polen zwingen, Flüchtlinge aufzunehmen.

Die polnischen Bauern erhalten geringere Zuschüsse als die Bauern anderer EU-Länder, daher können sie mit ihnen nicht auf gleicher Grundlage konkurrieren.

Die Pläne der EU, aus der Kohleverstromung auszusteigen, treiben die polnische Wirtschaft in eine Krise.

Die Arbeitsmigration von Polinnen und Polen in andere EU-Länder hat zur Folge, dass sich Polen entvölkert und Arbeitskräfte fehlen.

Die Europäische Union versucht, Polen Werte aufzudrängen, die im Widerspruch zur polnischen Tradition und Kultur stehen.

Die Notwendigkeit, dass die polnische Regierung das Recht der Europäischen Union respektieren muss, beschränkt die Souveränität Polens übermäßig.

Bürger anderer EU-Staaten können in Polen eine Arbeit aufnehmen und nehmen den Polen dadurch Arbeit weg.

Die Kosten der EU-Mitgliedschaft übersteigen die finanziellen Vorteile.

Quelle: Kucharczyk, Jacek: Widmo Polexitu? Społeczne postawy wobec członkostwa Polski w Unii Europejskiej [Das Gespenst des Polexit? Einstellungen der Gesellschaft gegenüber der Mitgliedschaft Polens in der Europäischen Union]. Warszawa: Instytut Spraw Publicznych (ISP) 2021. https://www.isp. org.pl/ (abgerufen am 01.02.2022). 
Grafik 4: Wie sehr vertrauen Sie den folgenden Institutionen? (Skala von 1 bis 5, Okt. 2021)

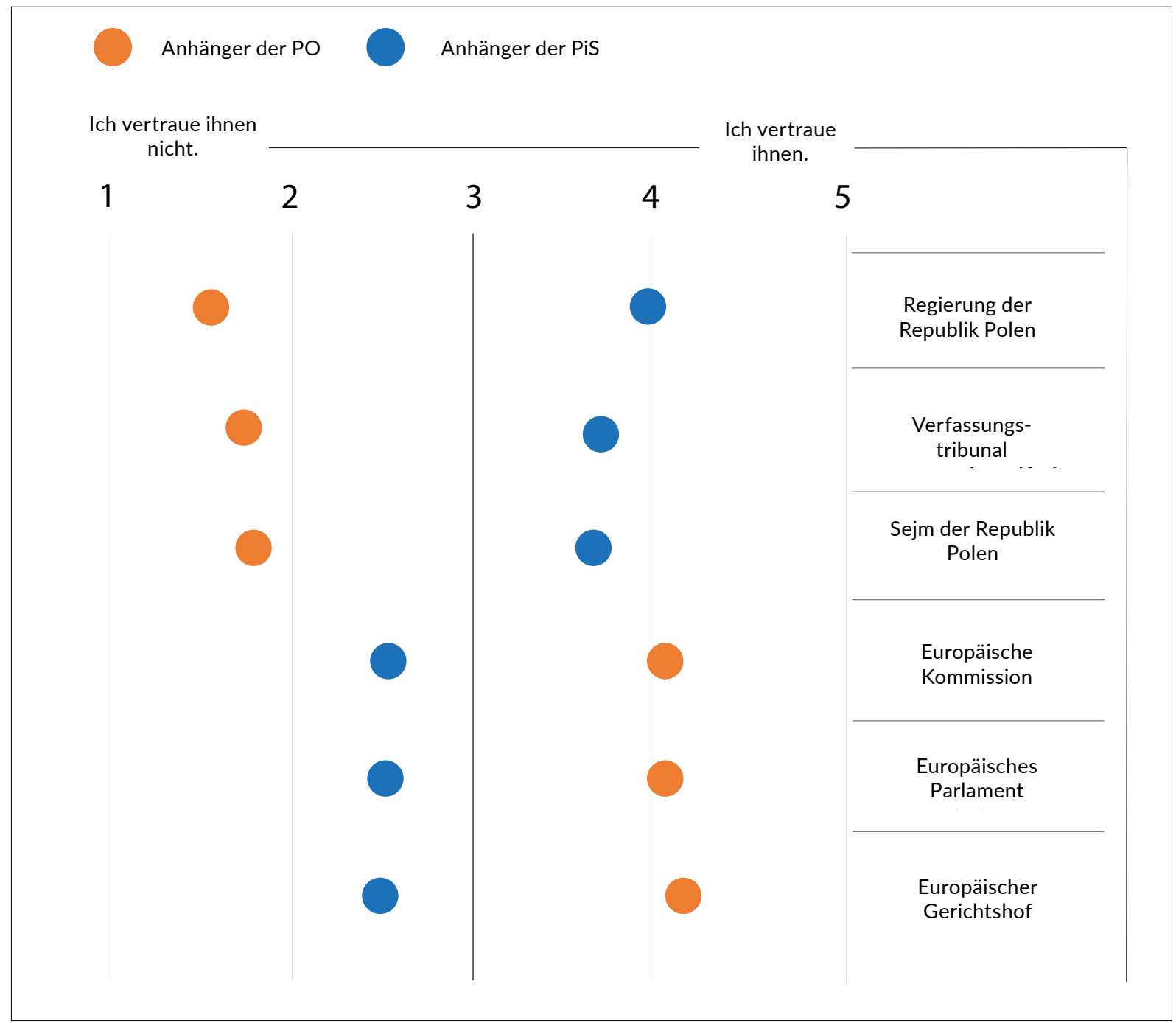

Quelle: Kucharczyk, Jacek: Widmo Polexitu? Społeczne postawy wobec członkostwa Polski w Unii Europejskiej [Das Gespenst des Polexit? Einstellungen der Gesellschaft gegenüber der Mitgliedschaft Polens in der Europäischen Union]. Warszawa: Instytut Spraw Publicznych (ISP) 2021. https://www.isp. org.pl/ (abgerufen am 01.02.2022).

Grafik 5: Stimmen Sie zu/nicht zu, dass die EU-Institutionen zu spät und zu schwach auf Verletzungen des EURechtes vonseiten der Regierungen der EU-Mitgliedsstaaten reagieren? (\%, Okt. 2021)

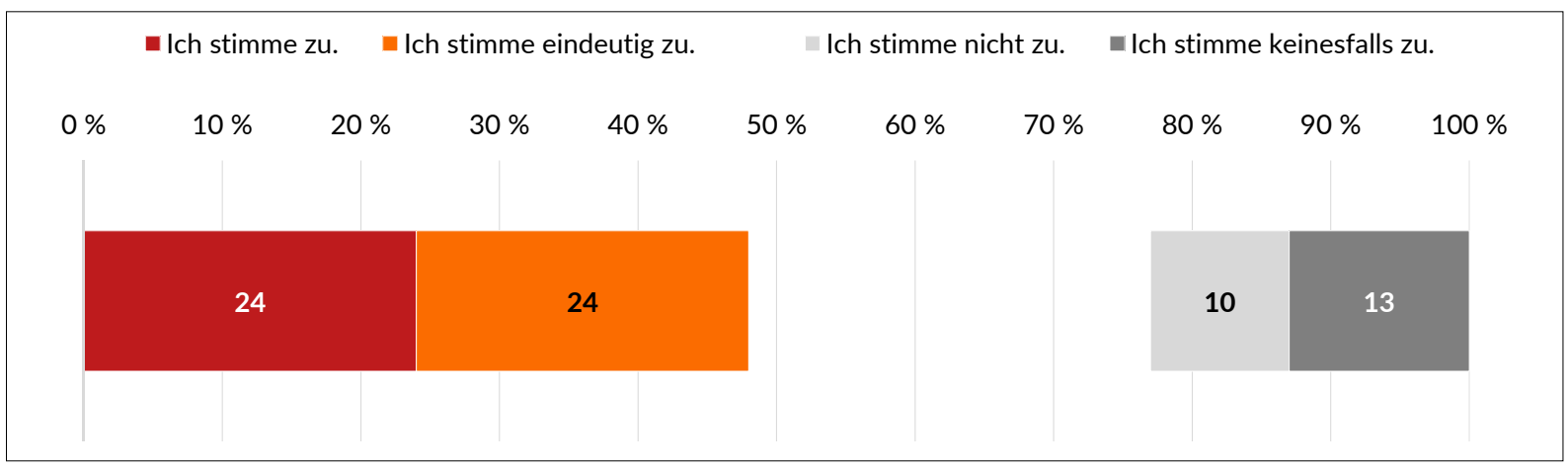

Quelle: Kucharczyk, Jacek: Widmo Polexitu? Społeczne postawy wobec członkostwa Polski w Unii Europejskiej [Das Gespenst des Polexit? Einstellungen der Gesellschaft gegenüber der Mitgliedschaft Polens in der Europäischen Union]. Warszawa: Instytut Spraw Publicznych (ISP) 2021. https://www.isp. org.pl/ (abgerufen am 01.02.2022). 
Grafik 6: Die Europäische Union hat die Regel eingeführt, dass die Auszahlung der EU-Fonds an die Mitgliedsländer von der Einhaltung des EU-Rechtes durch die Länder abhängig gemacht wird. Unterstützen Sie die Einführung dieser Regel? (\%, Okt. 2021)

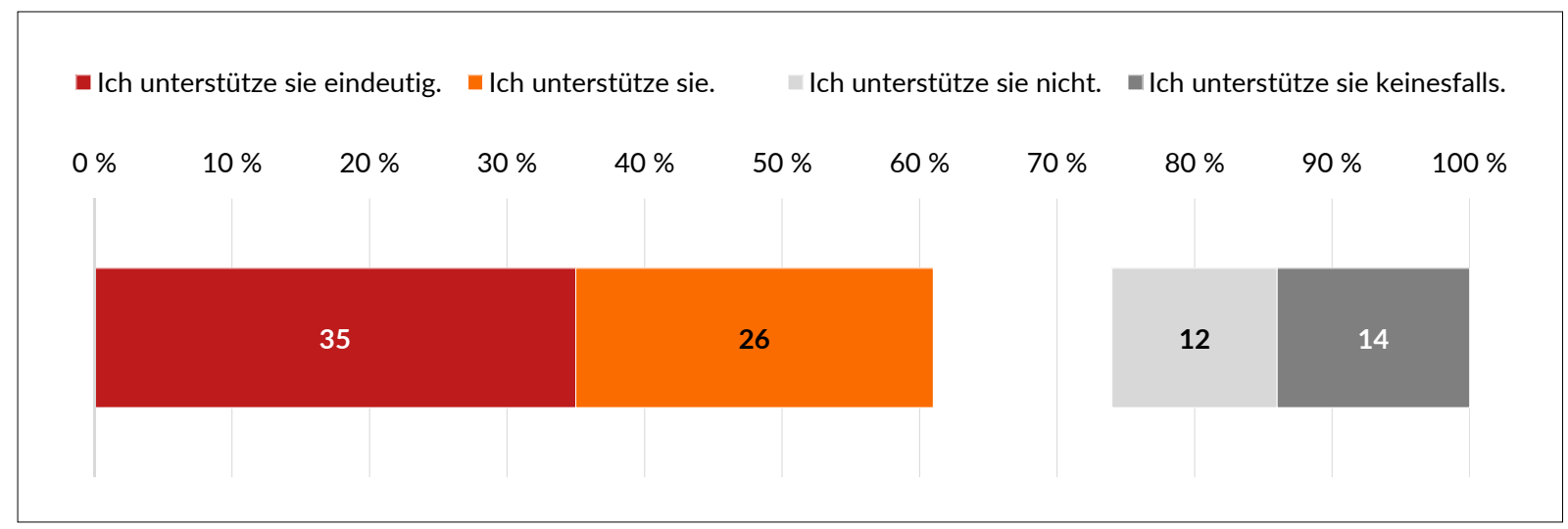

Quelle: Kucharczyk, Jacek: Widmo Polexitu? Społeczne postawy wobec członkostwa Polski w Unii Europejskiej [Das Gespenst des Polexit? Einstellungen der Gesellschaft gegenüber der Mitgliedschaft Polens in der Europäischen Union]. Warszawa: Instytut Spraw Publicznych (ISP) 2021. https://www.isp. org.pl/ (abgerufen am 01.02.2022)

\section{Grafik 7: Wie sehr vertrauen Sie den folgenden Institutionen? (\%, Okt. 2021)}

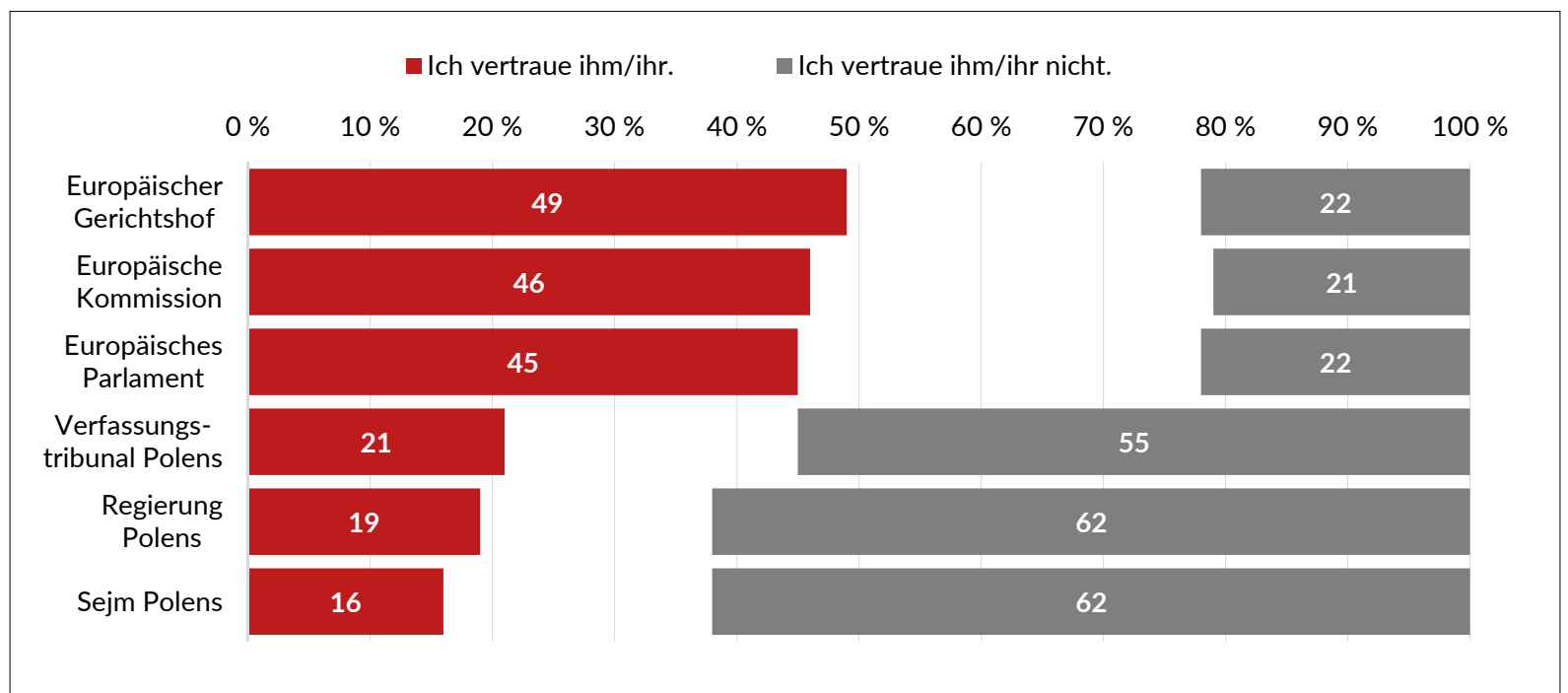

Quelle: Kucharczyk, Jacek: Widmo Polexitu? Społeczne postawy wobec członkostwa Polski w Unii Europejskiej [Das Gespenst des Polexit? Einstellungen der Gesellschaft gegenüber der Mitgliedschaft Polens in der Europäischen Union]. Warszawa: Instytut Spraw Publicznych (ISP) 2021. https://www.isp. org.pl/ (abgerufen am 01.02.2022). 
Grafik 8: Wird der gegenwärtige Zustand der Beziehungen zwischen der polnischen Regierung und den EUInstitutionen zu einem Austritt Polens aus der Europäischen Union führen? (\%, Okt. 2021)

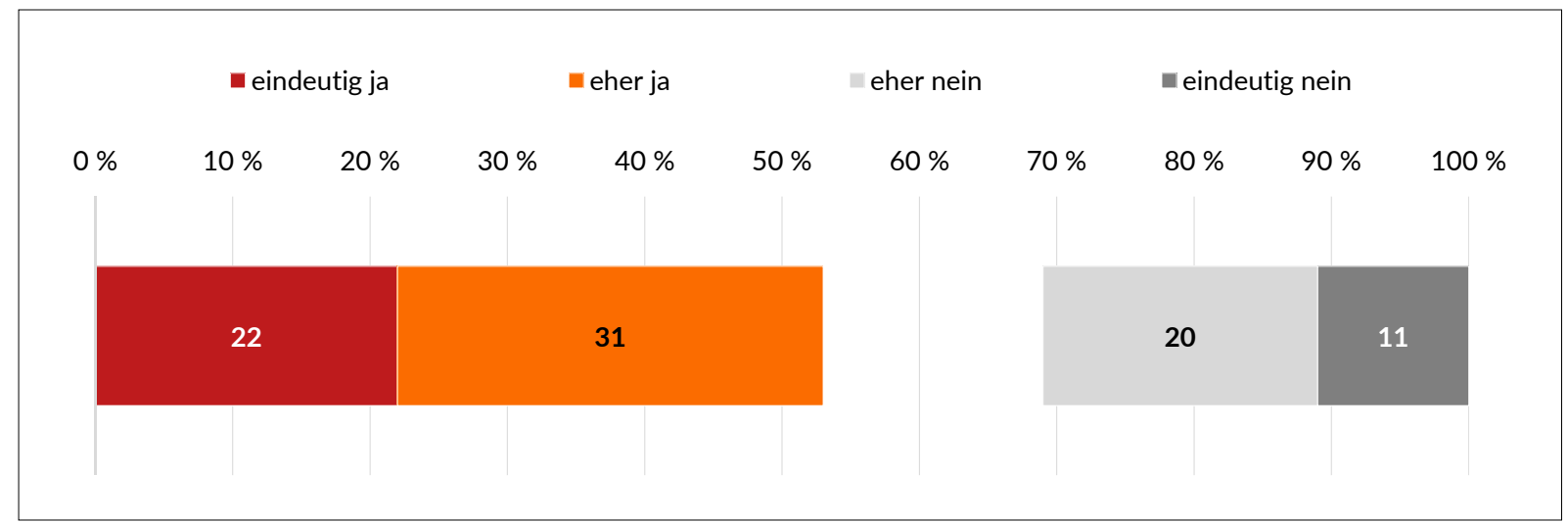

Quelle: Kucharczyk, Jacek: Widmo Polexitu? Społeczne postawy wobec członkostwa Polski w Unii Europejskiej [Das Gespenst des Polexit? Einstellungen der Gesellschaft gegenüber der Mitgliedschaft Polens in der Europäischen Union]. Warszawa: Instytut Spraw Publicznych (ISP) 2021. https://www.isp. org.pl/ (abgerufen am 01.02.2022).

Grafik 9: Welche Seite ist für den sich verschärfenden Konflikt zwischen der polnischen Regierung und den Institutionen der Europäischen Union verantwortlich? (\%, Okt. 2021)

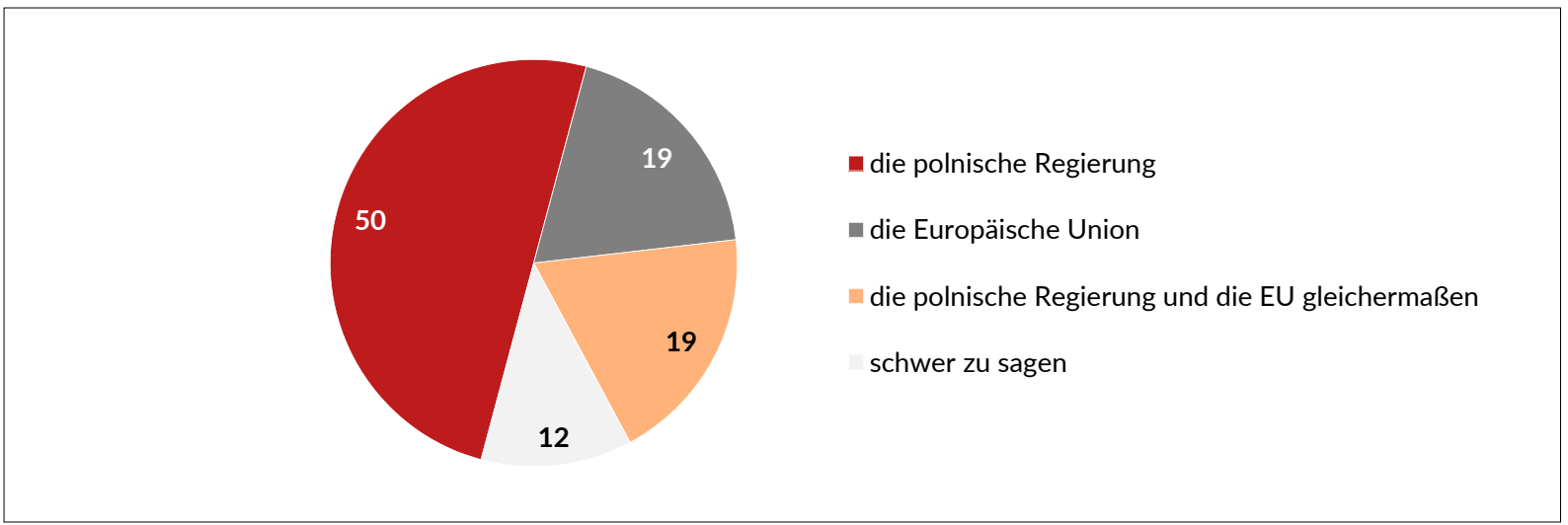

Quelle: Kucharczyk, Jacek: Widmo Polexitu? Społeczne postawy wobec członkostwa Polski w Unii Europejskiej [Das Gespenst des Polexit? Einstellungen der Gesellschaft gegenüber der Mitgliedschaft Polens in der Europäischen Union]. Warszawa: Instytut Spraw Publicznych (ISP) 2021. https://www.isp. org.pl/ (abgerufen am 01.02.2022). 
Grafik 10: Wie würde sich ein Polexit auf Polen und die polnische Gesellschaft in den genannten Bereichen auswirken? (\%, Okt. 2021)

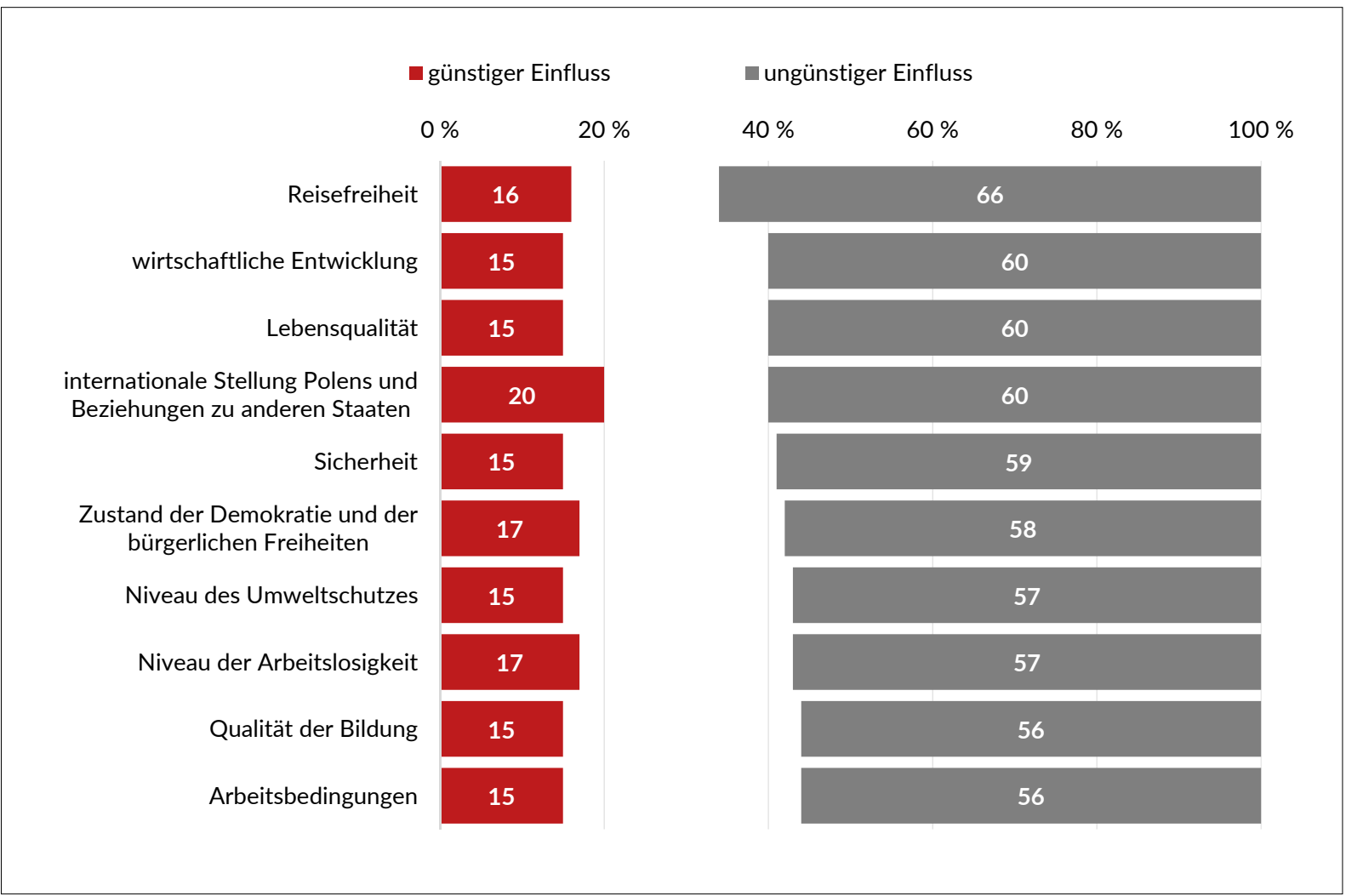

Quelle: Kucharczyk, Jacek: Widmo Polexitu? Społeczne postawy wobec członkostwa Polski w Unii Europejskiej [Das Gespenst des Polexit? Einstellungen der Gesellschaft gegenüber der Mitgliedschaft Polens in der Europäischen Union]. Warszawa: Instytut Spraw Publicznych (ISP) 2021. https://www.isp. org.pl/ (abgerufen am 01.02.2022).

Grafik 11: Wofür würden Sie bei einem eventuellen Referendum zum Austritt Polens aus der EU stimmen? (\%, Okt. 2021)

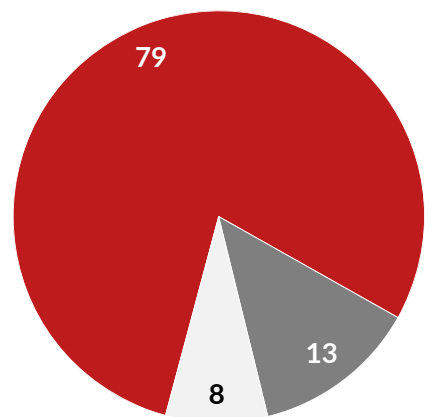

- für den Verbleib Polens in der EU

für den Austritt Polens aus der EU

schwer zu sagen

Quelle: Kucharczyk, Jacek: Widmo Polexitu? Społeczne postawy wobec członkostwa Polski w Unii Europejskiej [Das Gespenst des Polexit? Einstellungen der Gesellschaft gegenüber der Mitgliedschaft Polens in der Europäischen Union]. Warszawa: Instytut Spraw Publicznych (ISP) 2021. https://www.isp. org.pl/ (abgerufen am 01.02.2022). 
Grafik 12: Welche der folgenden Äußerungen spiegelt am besten Ihre Vorstellung über die Zukunft der EU wider? (Deutschland, Frankreich, Polen, Aug. 2021, \%)

- Um die eigene Souveränität besser zu schützen, soll Deutschland mit anderen Mitgliedsstaaten zusammenarbeiten, damit die EU-Institutionen einen Teil ihrer Kompetenzen wieder an die nationalen Regierungen abgeben.

- Deutschland und andere Mitgliedsstaaten sollen mehr Macht an die EU-Institutionen abgeben, damit die EU gemeinsame Herausforderungen Europas effektiver bewältigen kann.

schwer zu sagen

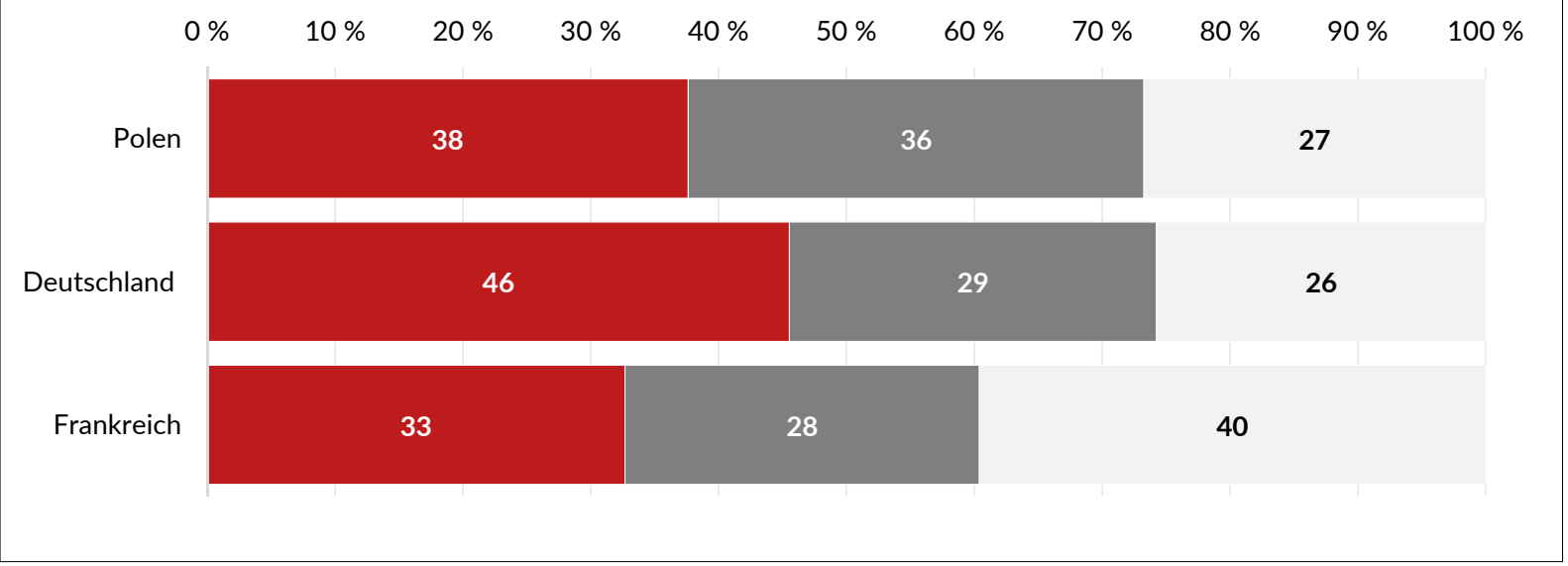

Quelle: Kucharczyk, Jacek: Mit Respekt für die Vergangenheit, mit Mut für die Zukunft. Deutsche, polnische und französische Wahrnehmungen des Weimarer Dreiecks und seiner Rolle in der EU. Warszawa: Instytut Spraw Publicznych 2021. www.isp.org.pl

\section{CHRONIK}

\section{1. - 14. Februar 2022}

\begin{tabular}{|l|l|}
\hline 01.02.2022 & $\begin{array}{l}\text { Ministerpräsident Mateusz Morawiecki trifft sich in Kiew (Ukraine) mit seinem ukrainischen Amtskollegen } \\
\text { Denys Schmyhal sowie mit dem ukrainischen Präsidenten Wolodymyr Selenskyj. Angesichts des russischen } \\
\text { Truppenaufmarsches von ca. 120.000 Soldaten an der russisch-ukrainischen Grenze bestätigt Morawiecki, } \\
\text { dass Polen der Ukraine Defensivwaffen liefern wird, u. a. Artilleriemunition, Mörser und Aufklärungsdroh- } \\
\text { nen. Thematisiert wird auch der Ausbau der Energieinfrastruktur zwischen beiden Ländern, um den Umfang } \\
\text { von Gaslieferungen aus dem Westen in die Ukraine zu erhöhen. Dies könne über die polnischen Terminals } \\
\text { für Flüssiggas (LNG) vonstattengehen. So könnten mögliche Reduzierungen russischer Gaslieferungen an die } \\
\text { Ukraine ausgeglichen werden. }\end{array}$ \\
\hline 02.02 .2022 & $\begin{array}{l}\text { Das US-Verteidigungsministerium gibt bekannt, dass es in den nächsten Tagen 1.700 Soldaten nach Polen ent- } \\
\text { senden wird sowie 1.000 nach Rumänien und 300 nach Deutschland. Hintergrund ist die russisch-ukrainisc- } \\
\text { he Krise. Russland hat ca. 120.000 Soldaten an der ukrainischen Grenze - sowie in Belarus im Rahmen eines }\end{array}$ \\
$\begin{array}{l}\text { Militärmanövers - zusammengezogen; die Ukraine, die NATO, die Europäische Union und die USA schlie- } \\
\text { ßen eine russische Invasion in die Ukraine nicht aus. }\end{array}$ \\
\hline $\begin{array}{l}\text { Außenminister Zbigniew Rau warnt bei einer Anhörung vor dem Helsinki-Ausschuss des US-amerikanischen } \\
\text { Kongresses (Ausschuss für Sicherheit und Zusammenarbeit in Europa) davor, dass sich der russisch-ukraini- } \\
\text { sche Konflikt auch auf Polen und weitere Staaten ausdehnen kann. Rau bestätigt damit die Einschätzung des } \\
\text { US-Kongressabgeordneten Steve Cohen (Demokratische Partei). Für eine solche Entwicklung sprächen viele } \\
\text { militärische und vor allem historische Argumente. Allerdings könne die Geschlossenheit der internationalen } \\
\text { Gemeinschaft dem Konflikt Einhalt gebieten. }\end{array}$ \\
\hline
\end{tabular}




\begin{tabular}{|c|c|}
\hline 04.02 .2022 & $\begin{array}{l}\text { Ein Gesetzesentwurf von Präsident Andrzej Duda, der die Auflösung der Disziplinarkammer am Obersten } \\
\text { Gericht (Sąd Najwyższy - SN) und stattdessen die Berufung einer Kammer für Berufliche Verantwortung vor- } \\
\text { sieht, wird an den Sejm geleitet. Duda reagiert damit auf das Urteil des Europäischen Gerichtshofes (EuGH) } \\
\text { vom Juli 2021, dass die Tätigkeit der Disziplinarkammer nicht EU-rechtskonform ist. }\end{array}$ \\
\hline 06.02 .2022 & $\begin{array}{l}\text { Präsident Andrzej Duda wird in Peking (Volksrepublik China) von seinem chinesischen Amtskollegen Xi Jin- } \\
\text { ping empfangen. Thematisiert wird der russische Truppenaufmarsch an der russisch-ukrainischen Grenze. Duda } \\
\text { sagt, ein eventueller Angriff Russlands auf die Ukraine würde den chinesischen Export nach Europa negativ } \\
\text { beeinflussen. Beide Staatsoberhäupter unterstreichen die Bedeutung der Charta der Vereinten Nationen für die } \\
\text { internationalen Beziehungen. Im Rahmen seines Arbeitsbesuches in China hat Duda zuvor an der Eröffnung } \\
\text { der 24. Olympischen Winterspiele in China teilgenommen. }\end{array}$ \\
\hline 07.02 .2022 & $\begin{array}{l}\text { Präsident Andrzej Duda zeigt sich zufrieden über die Einladung Deutschlands an das Weimarer Dreieck } \\
\text { (Deutschland, Frankreich, Polen) zu einem Treffen in Berlin am nächsten Tag. Er habe sich in seiner Amtszeit } \\
\text { seit sechs Jahren um eine Wiederaufnahme dieses Formates auf Ebene der Staats- und Regierungschefs bemüht. } \\
\text { Bei dem Treffen soll es um die Sicherheitslage an der russisch-ukrainischen Grenze gehen. }\end{array}$ \\
\hline 08.02 & $\begin{array}{l}\text { In Berlin treffen sich die Spitzen des Weimarer Dreiecks (Deutschland, Frankreich, Polen), Bundeskanzler } \\
\text { Olaf Scholz, Staatspräsident Emmanuel Macron und Präsident Andrzej Duda. In einer gemeinsamen Erklä- } \\
\text { rung rufen sie Russland zur Deeskalation an der russisch-ukrainischen Grenze und zu einem konstruktiven } \\
\text { Dialog über die Sicherheit auf dem europäischen Kontinent auf. Weitere militärische Aggressionen Russlands } \\
\text { gegenüber der Ukraine hätten ernste Konsequenzen. Sie sprechen sich für eine diplomatische Lösung des Kon- } \\
\text { fliktes aus und unterstützen das »Normandie-Format« und das Minsker Abkommen. }\end{array}$ \\
\hline 09.02 .2022 & $\begin{array}{l}\text { Łukasz Kohut, Europaabgeordneter der Linken (Lewica) in der Fraktion der Progressiven Allianz der Sozial- } \\
\text { demokraten, teilt mit, dass er eine Resolution für das Europäische Parlament vorbereitet hat, in der das polni- } \\
\text { sche Parlament und die Regierung aufgerufen werden, Schlesisch als Regionalsprache anzuerkennen. Aktuell } \\
\text { hat Kaschubisch den Status einer Regionalsprache in Polen. }\end{array}$ \\
\hline 10.02 & $\begin{array}{l}\text { Der Abgeordnetenkreis von Paweł Kukiz reicht bei Sejmmarschallin Elżbieta Witek einen Antrag auf Berufung } \\
\text { eines Untersuchungsausschusses zur Klärung des Einsatzes der Überwachungssoftware Pegasus in den Jah- } \\
\text { ren } 2005 \text { bis } 2021 \text { gegenüber Oppositionspolitikern und Akteuren, die der Opposition nahestehen, ein. Den } \\
\text { Antrag haben auch Abgeordnete der Bürgerkoalition (Koalicja Obywatelska - KO), der Linken (Lewica), der } \\
\text { Polnischen Koalition - Polnische Bauernpartei (Koalicja Polska - Polskie Stronnictwo Ludowe/PSL), der Pol- } \\
\text { nischen Sozialistischen Partei (Polska Partia Socjalistyczna - PPS), von Verständigung (Porozumienie) sowie } \\
\text { der Konföderation (Konfederacja) unterzeichnet. }\end{array}$ \\
\hline 11.02 & $\begin{array}{l}\text { Die Fraktion von Recht und Gerechtigkeit (Prawo i Sprawiedliwość - PiS) reicht im Sejm einen Gesetzesent- } \\
\text { wurf ein, der vorsieht, dass das Oberste Gericht (Sąd Najwyższy - SN) Disziplinarangelegenheiten bei Rich- } \\
\text { tern behandeln soll. Die dafür eingerichtete Disziplinarkammer beim SN, deren Auflösung der Europäische } \\
\text { Gerichtshof (EuGH) im Juli } 2021 \text { angeordnet hat, soll bestehen bleiben, sich aber nicht mit Disziplinarfällen } \\
\text { bei Richtern, sondern anderen juristischen Berufsgruppen befassen. Dem Vernehmen nach wurde der Geset- } \\
\text { zesentwurf vorher weder mit Präsident Andrzej Duda, der vor einer Woche ebenfalls ein Gesetzesprojekt als } \\
\text { Reaktion auf das EuGH-Urteil eingereicht hatte, noch mit dem Koalitionspartner Solidarisches Polen (Soli- } \\
\text { darna Polska), der den Justizminister und Generalstaatsanwalt Zbiegniew Ziobro stellt, konsultiert. }\end{array}$ \\
\hline 11.02 .2022 & $\begin{array}{l}\text { Das US-amerikanische Verteidigungsministerium gibt bekannt, dass es nach Polen zusätzlich } 3.000 \text { Solda- } \\
\text { ten, die bisher nicht in Europa stationiert sind, entsendet. Hintergrund ist der russische Truppenaufmarsch an } \\
\text { der ukrainischen Ostgrenze sowie ein russisch-belarussisches Militärmanöver und die Sorge vor einem Ein- } \\
\text { marsch in die Ukraine. }\end{array}$ \\
\hline 14.02 .2022 & $\begin{array}{l}\text { Die Sprecherin der Kreisstaatsanwaltschaft in Grünberg (Zielona Góra), Ewa Antonowicz, teilt mit, dass gegen } \\
\text { zehn Personen, darunter eine deutsche Staatsbürgerin, Anklage wegen Gewalt gegen Polizeibeamte erhoben } \\
\text { wird. Zu den Festnahmen kam es vor zwei Tagen, als ca. } 100 \text { Personen nach einem Aufruf der Gruppe No Bor- } \\
\text { ders Team unter dem Slogan »Genug mit der Kriminalisierung von Migranten«in Krosno Odrzańskie demons- } \\
\text { trierten. Dabei wurden Polizisten mit Steinen und Pyrotechnik angegriffen. Die Polizei setzte Tränengas ein. } \\
\text { Nach Angaben der Polizei war die Versammlung illegal. }\end{array}$ \\
\hline
\end{tabular}

Sie können die gesamte Chronik seit 2007 auch auf http://www.laender-analysen.de/polen/ unter dem Link "Chronik« lesen. 
Die Polen-Analysen erscheinen zweimal monatlich als E-Mail-Dienst. Sie werden gemeinsam vom Deutschen PolenInstitut Darmstadt, der Forschungsstelle Osteuropa an der Universität Bremen und weiteren Partnern eines Konsortiums (siehe Titelseite) herausgegeben.

Ein Archiv der Polen-Analysen finden Sie im Internet unter www.laender-analysen.de/polen Kostenloses Abonnement unter http://www.deutsches-polen-institut.de/Newsletter/subscribe.php

Diese Analysen finden Sie online als Lizenzausgabe auf

bpb.de

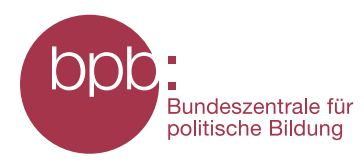

Deutsches Polen-Institut Darmstadt (www.deutsches-polen-institut.de)

Das seit 1980 tätige Deutsche Polen-Institut Darmstadt (DPI) ist ein Forschungs-, Informations- und Veranstaltungszentrum für polnische Kultur, Geschichte, Politik, Gesellschaft und die deutsch-polnischen Beziehungen, die sich im Kontext der europäischen Integration entwickeln. Institutionelle Träger des DPI sind das Land Hessen, die Kultusminister der Länder, das Auswärtige Amt und die Wissenschaftsstadt Darmstadt. Einen wesentlichen Beitrag zur Verwirklichung der Institutsziele leisten private Stiftungen. Ziel der Vermittlertätigkeit des DPI ist es, »die zu interessieren, auf die es politisch, wirtschaftlich, gesellschaftlich und kulturell im deutsch-polnischen Verhältnis ankommt (Leitlinien 1997). Es geht um die Entscheider und Multiplikatoren in Politik, Kultur, Bildung, Verwaltung, Medien und Wirtschaft. Das DPI versteht sich in Kooperation mit den Orten wissenschaftlicher Polen-Kompetenz an deutschen Hochschulen und Forschungsinstituten als verbindendes und vernetzendes Zentrum. Mit der 70.000 Bände zählenden multidisziplinären Fachbibliothek für Polen, die eine einzigartige Sammlung polnischer Belletristik in der Originalsprache und in deutscher Übersetzung umfasst, ist das DPI ein geschätzter Ort der Recherche und des wissenschaftlichen Arbeitens.

Forschungsstelle Osteuropa an der Universität Bremen (www.forschungsstelle.uni-bremen.de)

1982 gegründet, widmet sich die Forschungsstelle Osteuropa an der Universität Bremen der interdisziplinären Analyse der Länder Ost- und Ostmitteleuropas in Zeitgeschichte und Gegenwart. Der Forschungsschwerpunkt liegt dabei auf der Rolle von "Dissens und Konsens«, von Opposition und Zivilgesellschaft in ihrem historischen, politischen, gesellschaftlichen und kulturellen Kontext. Die Forschungsstelle besitzt in ihrem Archiv eine einzigartige Sammlung alternativer Kulturgüter und unabhängiger Texte aus den ehemaligen sozialistischen Ländern. Darunter befindet sich auch eine umfangreiche Sammlung des "Zweiten Umlaufs«, die das Schrifttum und Dokumente unabhängiger Initiativen und gesellschaftlicher Gruppen in Polen aus der Zeit von 1976 bis zum Umbruch umfasst. Hinzu kommt eine umfangreiche Bibliothek mit wissenschaftlicher Literatur. Mit Archiv, Bibliothek und zwei wissenschaftlichen Abteilungen ist die Forschungsstelle auch eine Anlaufstelle sowohl für Gastwissenschaftler als auch für die interessierte Öffentlichkeit.

Eine der Hauptaufgaben der Forschungsstelle ist die Information der interessierten Öffentlichkeit. Dazu gehören unter anderem regelmäßige E-Mail-Informationsdienste für Politik, Wirtschaft, Zivilgesellschaft und Medien.

Herausgeber:

Deutsches Polen-Institut, Deutsche Gesellschaft für Osteuropakunde e.V., Forschungsstelle Osteuropa an der Universität Bremen, Leibniz-Institut für Agrarentwicklung in Transformationsökonomien, Leibniz-Institut für Ost- und Südosteuropaforschung, Zentrum für Osteuropa- und internationale Studien (ZOiS) gGmbH

Redaktion:

Dr. Andrzej Kaluza (verantwortlich) (Darmstadt) und Dr. Silke Plate (Bremen)

Satz: Matthias Neumann

Wissenschaftlicher Beirat:

Prof. Dr. Stefan Garsztecki, Technische Universität Chemnitz

Prof. Dr. Klaus Ziemer, Kardinal-Stefan-Wyszyński-Universität Warschau

Die Meinungen, die in den Polen-Analysen geäußert werden, geben ausschließlich die Auffassung der Autoren wieder.

Abdruck und sonstige publizistische Nutzung sind nach Rücksprache mit der Redaktion gestattet. Polen-Analysen-Layout:Cengiz Kibaroglu, Matthias Neumann

Alle Ausgaben der Polen-Analysen sind mit Themen- und Autorenindex archiviert unter www.laender-analysen.de

ISSN 1863-9712 @ 2022 by Deutsches Polen-Institut, Deutsche Gesellschaft für Osteuropakunde e.V., Forschungsstelle Osteuropa an der Universität Bremen, Leibniz-Institut für Agrarentwicklung in Transformationsökonomien, Leibniz-Institut für Ost- und Südosteuropaforschung, Zentrum für Osteuropa- und internationale Studien (Z0iS) gGmbH

Kontakt: Dr. Andrzej Kaluza, Deutsches Polen-Institut, Residenzschloss, Marktplatz 15, 64283 Darmstadt,

Tel.: +49/6151/4202-20, Fax: +49/6151/4202-10, E-Mail: kaluza@dpi-da.de, Internet: www.laender-analysen.de/polen 


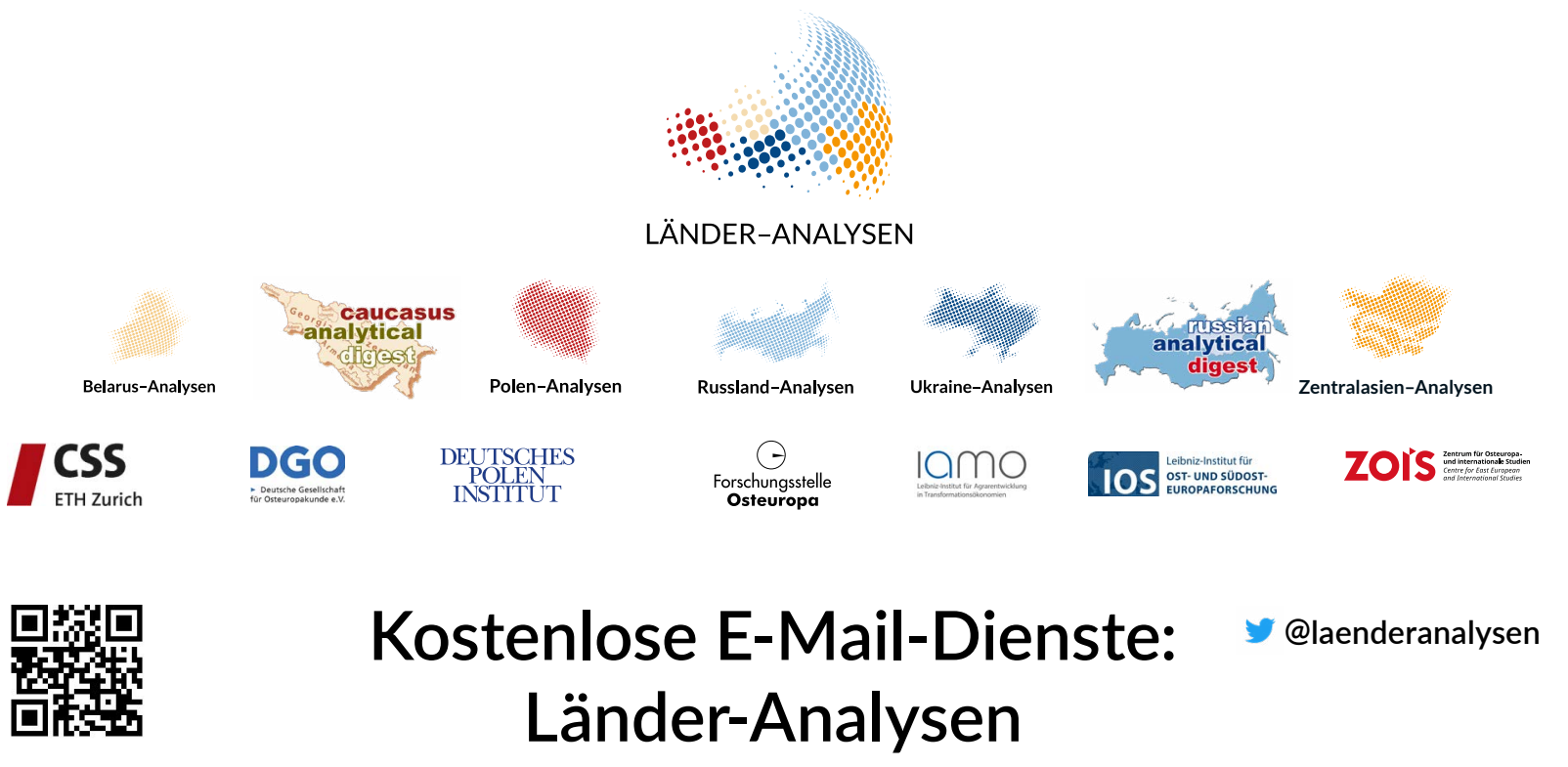

Die Länder-Analysen bieten regelmäßig im kostenlosen Abonnement kompetente Einschätzungen aktueller politischer, wirtschaftlicher, sozialer und kultureller Entwicklungen in Ostmitteleuropa und der GUS. Alle Länder-Analysen verstehen sich als Teil eines gemeinsamen Projektes, das der wissenschaftlich fundierten, allgemeinverständlich formulierten Analyse der Entwicklungen im östlichen Europa, der Offenheit für verschiedene inhaltliche Positionen und der kostenlosen und nicht-kommerziellen Information einer breit verstandenen interessierten Öffentlichkeit verpflichtet ist. Autor/innen sind internationale Fachwissenschaftler/innen und Expert/innen. Die Redaktionen der Länder-Analysen bestehen aus Wissenschaftler/innen mit langjähriger Forschungserfahrung.

Die deutschsprachigen Länder-Analysen werden gemeinsam von der Forschungsstelle Osteuropa an der Universität Bremen, dem Zentrum für Osteuropa- und internationale Studien, der Deutschen Gesellschaft für Osteuropakunde, dem Deutschen Polen-Institut, dem Leibniz-Institut für Agrarentwicklung in Transformationsökonomien und dem Leibniz-Institut für Ost- und Südosteuropaforschung herausgegeben. Die englischsprachigen Länder-Analysen erscheinen in Kooperation der Forschungsstelle Osteuropa mit dem Center for Security Studies (CSS) der ETH Zürich.

Die Länder-Analysen bieten regelmäßig Kurzanalysen zu aktuellen Themen, ergänzt um Grafiken und Tabellen sowie Dokumentationen. Zusätzlich gibt es eine Chronik aktueller Ereignisse.

\section{Belarus-Analysen}

Erscheinungsweise: zweimonatlich

Abonnement unter: http://www.laender-analysen.de/belarus/

\section{Caucasus Analytical Digest}

In englischer Sprache. Erscheinungsweise: zweimonatlich

Abonnement unter: http://www.css.ethz.ch/en/publications/cad.html

\section{Polen-Analysen}

Erscheinungsweise: zweimal monatlich

Abonnement unter: http://www.deutsches-polen-institut.de/newsletter/polen-analysen/

\section{Russland-Analysen}

Erscheinungsweise: zweimal monatlich

Abonnement unter: http://www.laender-analysen.de/russland/

\section{Russian Analytical Digest}

In englischer Sprache. Erscheinungsweise: zweimal monatlich Abonnement unter: http://www.css.ethz.ch/en/publications/rad.html

\section{Ukraine-Analysen}

Erscheinungsweise: zweimal monatlich

Abonnement unter: http://www.laender-analysen.de/ukraine/

\section{Zentralasien-Analysen}

Erscheinungsweise: zweimonatlich

Abonnement unter: http://www.laender-analysen.de/zentralasien/ 